\title{
THE DIRAC OPERATOR ON SPACES WITH CONICAL SINGULARITIES AND POSITIVE SCALAR CURVATURES ${ }^{1}$
}

BY

\author{
ARTHUR WEICHUNG $\mathrm{CHOU}^{2}$
}

\begin{abstract}
We study, in the spirit of Jeff Cheeger, the Dirac operator on a space with conical singularities. We obtain a Bochner-type vanishing theorem and prove an index theorem in the singular case. Also, the relationship with manifolds with boundary is explored. In the Appendix two methods of deforming the metric near the boundary are established and applied to obtain several new results on constructing complete metrics with positive scalar curvature.
\end{abstract}

0. Introduction. In recent years analytic tools (e.g., elliptic operator theory) have become increasingly important in studying the topology of compact smooth manifolds. Examples are Bochner's method and the Atiyah-Singer index theorem.

Bochner's method is to obtain a local formula expressing a geometric (elliptic) operator as the sum of a "rough Laplacian", which is nonnegative on closed manifolds, and a curvature term. Thus, if the curvature term is positive, the kernel of this operator vanishes. Results of this type are called "vanishing theorems". For the Dirac operator $D$ on spinors, we have the Lichnerowicz-Bochner formula

$$
D^{2}=\nabla^{*} \circ \nabla+\kappa / 4,
$$

where $\nabla$ is the connection on the bundle of spinors and $\kappa$ is the scalar curvature. Again for closed manifolds, $\kappa>0$ yields $\operatorname{Ker}\left(D^{2}\right)=\{0\}$, i.e., there are no harmonic spinors (see [22, 23]).

The Atiyah-Singer index theorem [1, 4] says that, on a closed manifold the index of an elliptic operator $L$, i.e., $\operatorname{dim}(\operatorname{Ker}(L))-\operatorname{dim}(\operatorname{Coker}(L))$, can be expressed as a linear combination of Pontrjagin numbers which are toplogical invariants. For the case of the Dirac operator $D$ and an even-dimensional closed spin manifold $M$, Lichnerowicz [23] obtained from the index theorem that

$$
\text { Index }\left(D^{+}\right)=\hat{A}(M), \quad \text { the } \hat{A} \text {-genus of } M \text {. }
$$

Combined with the vanishing theorem (0.1), it follows that $\kappa>0$ implies $\hat{A}(M)=0$. This provides us with a topological obstruction to the existence of a metric on $M$ with $\kappa>0$. Recently, Gromov and Lawson have greatly extended the scope of this method by considering spinors with coefficients in suitable bundles, and they also developed a parallel theory for the Dirac operator on complete manifolds (see [16, 18]).

Received by the editors February 29, 1984 and, in revised form, June 18, 1984. 1980 Mathematics Subject Classification. Primary 58G11, 58G12, 58G25; Secondary 53C20.

${ }^{1}$ This research is supported in part by the National Science Foundation under Grant No. MCS-8301627.

${ }^{2}$ Part of this paper was written at the Institute for Advanced Study, and the author wishes to thank the Institute for its hospitality. 
An extension of this kind of theory to singular spaces for the Laplacian $\Delta$ was developed by Jeff Cheeger in [6-8] (see also [10, 11]). In this paper we establish the corresponding theory for the Dirac operator by use of his ideas. Let us not recall some definitions and basic ideas.

Let $N^{m}$ be a closed Riemannian manifold of dimension $m$ with metric $g$. By the cone $C(N)$ on $N$, we mean the space $(0, \infty) \times N$ equipped with the metric $d r \otimes d r$ $+r^{2} g$, where $r \in(0, \infty)$. Set

$$
C_{0, u}(N)=\{(r, x) \in C(N), 0<r<u\}
$$

and

$$
\overline{C_{0, u}(N)}=\{(r, x) \in C(N), 0 \leqslant r \leqslant u\} .
$$

(0.3) Definition. $X^{m+1}$ is called a space with conical singularities if there exists $P_{j} \in X^{m+1}, j=1,2, \ldots, k$, such that $X^{m+1} \backslash \bigcup_{j=1}^{k}\left\{P_{j}\right\}$ is a smooth Riemannian manifold, and each $P_{j}$ has a neighborhood $U_{j}$ such that $U_{j} \backslash\left\{P_{i}\right\}$ is isometric to $C_{0, u}\left(N_{j}^{m}\right)$ for some $u_{j}$ and $N_{j}^{m}$.

Without loss of generality we assume that $k=1$ and $u_{j} \geqslant 1$. We write $X^{m+1}$ $=\overline{C_{0,1}\left(N^{m}\right)} \cup M^{m+1}$, where $N=\partial M$ and the union is along the boundary. Of course, in general, $X^{m+1}$ is not a manifold. For the purpose of this paper we also assume that $X^{m+1} \backslash\{P\}$ is a spin manifold and $N^{m}$ has the induced spin structure (see $\$ 1$ for defintions). Notice that $C\left(S_{1}^{m}\right)=R^{m+1}$, where $S_{1}^{m}$ is the standard sphere of radius one. This is just Euclidean space in polar coordinates.

By definition, analysis on a space with conical singularities $X$ means analysis on the smooth part $X \backslash\{P\}$ of $X$. Since this manifold is incomplete, the situation is quite different from that of a compact or complete manifold. For example, the elliptic operators, such as the Laplacian and the Dirac operator, are no longer essentially selfadjoint. Thus we have to choose a particular selfadjoint extension. See $[6,7]$ for the case of the Laplacian.

Because local analysis on $M$ is well understood, we first restrict our attention to the cone part $C_{0,1}(N)$. To carry out analysis on the cone, we observe that, by using separation of variables, we can reduce local analysis on the cone to global analysis on the cross-section $N$. In fact, if we restrict a function $g(r, x)$ on $C(N)$ to $\{r\} \times N$, then by standard theory of eigenfunction expansion of the elliptic operator $L$ on compact manifolds, we can write

$$
g(r, x)=\sum g_{i}(r) \phi_{i}(x),
$$

where $\left\{\phi_{i}\right\}$ are the eigenfunctions of $\tilde{L}_{r}=L_{\mid\{r\} \times N}$ which can be identified with those of $\tilde{L}=L_{\mid\{1\} \times N}$ by (parallel) translation along the radial geodesic $R \times\{x\}$. The convergence of (0.4) is in the $L^{2}$ sense; moreover, if $g$ is smooth, then a standard argument shows that the convergence is uniform on each compact subset away from the singularity at $p$. Notice that in the case of $C\left(S_{1}^{m}\right)=R^{m+1}$, this is nothing but the usual Fourier series expansion.

On functions of the type $g(r) \phi(x)$, the action of $L$ will give us singular Sturm-Liouville ordinary differential equations (see (2.6)), and, hence, we can solve for the eigenfunctions of $L$ explicitly on the cone. As we will see, there are limit 
circle cases (see [28]) of singular Sturm-Liouville equations corresponding to the small eigenvalues of $L$ on $\{1\} \times N$. This is the reason why $L$ fails to be selfadjoint without further conditions on the behavior of the function near the singularity at $r=0$.

In $\$ 2$ we derive the separation of variables formula, write down the eigenspinors for the Dirac operator $D$ on the cone, and construct counterexamples to the selfadjointness of $D$. Then in $\S 3$ we obtain the criterion for the selfadjointness of $D$ from an a priori estimate. The main result is the following (compare [7]). Let $D_{0}$ denote the Dirac operator on the space of smooth spinors with compact support on $X=C_{0,1}\left(N^{m}\right) \cup M$, and let $\tilde{D}$ denote the Dirac operator on $\{1\} \times N=N$. Then we have

(3.2) TheOREM. The Dirac operator $D_{0}$ is essentially selfadjoint if and only if there is no eigenvalue $\mu_{j}$ of $\tilde{D}$ such that $\left|\mu_{j}\right|<1 / 2$.

Let $D$ denote the Dirac operator with domain $\left\{\phi \mid \phi \in L^{2} \cap C^{\infty}\right.$ and $\left.D \phi \in L^{2}\right\}$, and let $\bar{D}$ denote its $L^{2}$ closure. Let ${ }^{*}$ denote the $L^{2}$ dual of an operator. It follows that

$$
\bar{D}_{0}=\bar{D} \quad \text { and } \quad \bar{D}_{0}^{*} \cdot \bar{D} \quad \text { if }\left|\mu_{j}\right| \geqslant 1 / 2
$$

Let us write $\Delta_{D}=\bar{D}_{0}^{*} \cdot \bar{D}_{0}$ and $\Delta_{N}=\bar{D}^{*} \cdot \bar{D}$, which correspond to the generalized Dirichlet and Neumann conditions, respectively. Both $\Delta_{D}$ and $\Delta_{N}$ are selfadjoint extensions of $\Delta_{0}=\left(D_{0}\right)^{2}$. We should mention that even if $D_{0}$ is essentially selfadjoint, $\Delta_{0}$ may still not be essentially selfadjoint because of the limit circle phenomenon (see $[6,28])$.

In $\S 4$ we show that if $C_{0,1}(N)$ has nonnegative scalar curvature, then the condition $\left|\mu_{j}\right| \geqslant 1 / 2$ is automatically satisfied. This, together with $(0.1)$, gives us the vanishing theorem for spaces with conical singularities.

(4.2) THEOREM. If $X^{m+1}=C_{0,1}\left(N^{m}\right) \cup M$ has scalar curvature $\kappa \geqslant 0$ and $\kappa>0$ somewhere, then $\operatorname{Ker}(\bar{D})=\{0\}$.

In $\$ 5$ we combine this theorem with our index formulas to obtain topological conclusions. This theorem can also be construed as giving necessary conditions for a manifold with boundary to admit a metric with scalar curvature $\kappa \geqslant 0$ for which the metric near the boundary is conical.

A general discussion of manifolds with boundary is given in the Appendix to $\$ 4$. By use of certain deformation techniques, one of which is a generalization of the bending techinque in [16], we obtain the following theorems. Let $M$ be a manifold with boundary $N^{m}$. Let $\kappa$ denote the scalar curvature of $M$ and $\kappa_{r}$ the scalar curvature of the hypersurface at distance $r$ to the boundary.

THEOREM [A.2]. Suppose

(i) $\kappa \geqslant 0$,

(ii) $\kappa_{r} \geqslant 0 \forall r \in[0, \varepsilon]$,

(iii) $H_{r} \geqslant 0 \forall r \in[0, \varepsilon]$, where $H_{r}$ is the mean curvature w.r.t the exterior normal. 
Then in this neighborhood $[0, \varepsilon] \times N$ the metric can be deformed to a complete metric, which ends with the product metric $R \times N$ near infinity, with nonnegative scalar curvature.

This is similar to Theorem 5.7 in [16].

TheOREM [A.12]. Suppose $\operatorname{dim} N=m \geqslant 2$ and

(i) the tubular neighborhood of $N$ in $M$ is normalized to be of width 1 and $\kappa>16 m /(m+1)$ on it,

(ii) $\kappa_{r} \geqslant 0(>0)$ on $\{r\} \times N \forall r \in[0,1]$.

Then the metric can be deformed to a complete metric, which ends with the product metric $R \times N$ near infinity, with scalar curvature $\kappa^{\prime} \geqslant 0(>0)$.

The discussion in the Appendix shows that our vanishing theorem could also be obtained from Gromov and Lawson [18], except when $K \equiv 0$ on a conical neighborhood of the boundary. This case is not covered by their method.

The next step is to study index formulas. We follow the same procedure as in [6, 8], which is based on the functional calculus of the Laplacian on the cone and the heat equation method of deriving the index formula (see [1]). Let us briefly recall the ideas in [8]:

1. Using the technique of separation of variables, i.e., the eigenfunction expansion (0.4), and the Hankel transform, we can obtain a spectral representation of the Laplacian $\Delta$ on the cone $C(N)$ such that the action of $\Delta$ is carried into multiplication of $\lambda^{2}$; moreover, according to the Hankel inversion formula (5.5), the following formal representation for the kernel $f(\Delta)$ on $C(N) \times C(N)=\left\{\left(r_{1}, x_{1}, r_{2}, x_{2}\right)\right\}$ holds.

$$
\begin{aligned}
f(\Delta)=\left(r_{1} r_{2}\right)^{c} \sum_{j}\left(\int_{0}^{\infty} f\left(\lambda^{2}\right) J_{\nu_{j}}\left(\lambda r_{1}\right) J_{\nu_{j}}\left(\lambda r_{2}\right) \lambda d \lambda\right) \\
\phi_{j}\left(x_{1}\right) \otimes \phi_{j}\left(x_{2}\right),
\end{aligned}
$$

where $c=(1-m) / 2, \phi_{j}$ are the eigenfunctions of $\tilde{\Delta}$ (the Laplacian on $N$ ) with eigenvalue $\mu_{j}$, and $J_{\nu_{j}}$ is the Bessel function of order $\nu_{j}=\sqrt{c^{2}+\mu_{j}}$.

Thus we can regard (0.5) as the sum of series consisting of a family of functions of $\tilde{\Delta}$ on $N$ parametrized by $\left(r_{1}, r_{2}\right)$ in the distribution sense. Notice that in the case of $C\left(S_{1}^{m}\right)=R^{m+1}$, the Hankel transform is nothing but the Fourier transform in polar coordinates, and $(0.5)$ is just the representation of the kernel $f(\Delta)$ via the Fourier inversion formula.

2. Making use of the classical integral formulas of Bessel functions, we can explicitly integrate $(0.5)$ for certain functions $f$; e.g., the heat kernel $f(\Delta)=e^{-t \Delta}$ and the zeta function $f(\Delta)=\Gamma(s) \Delta^{-s}$ (see (5.9), (5.11)).

These explicit expressions, together with the property of conformal homogeneity of the cone, enable us to compute the asymptotic expansion of the trace of the heat kernel on the cone in terms of functions of $\tilde{\Delta}$ on $N^{m}$.

3. It follows from Duhamel's principle (see $[5,8]$ ) that a parametrix for the heat kernel on $X=C_{0,1}(N) \cup M$ can be obtained by gluing together the heat kernel on $M$ with the one on $C_{0,1}\left(N^{m}\right)$. Then, from the behavior of the heat kernel near the 
singularity at $r=0$, we can conclude that the heat kernel on $X$ is of trace class. This shows that Green's operator is compact, and, hence, Fredholm theory can be applied to obtain the standard global results as in the nonsingular case; e.g., the existence of a complete orthonormal basis of $L^{2}$ consisting of eigenfunctions (-forms) of $\Delta$ with discrete eigenvalues $0 \leqslant \lambda_{0} \leqslant \lambda_{1}, \ldots, \rightarrow \infty$.

4. In order to compute the index of the geometric operator on $X$, we apply the heat equation method, which says that the index is equal to the constant term in the asymptotic expansion of the trace of a certain modified heat kernel on $X^{m+1}$. As can easily be seen from the previous discussion, this constant term must consist of two separate terms; one is from the manifold $M$; the other, from the singular part $C_{0,1}(N)$. The first contribution is the integral of the same characteristic form over $M$ as in the nonsingular case by the Patodi-Gilkey theorem (see [1]). The second contribution from the singularity can be shown to be an eta-invariant of the manifold $N$ (see [3]).

In $\$ 5$ we carry out the above program for the Dirac operator and obtain the following index formula. Suppose that $X^{m+1}\{p\}$ is an even-dimensional spin manifold. Let $\bar{D}_{0}$ and $\bar{D}$ denote the Dirac operators as in (0.5). Let $\bar{D}_{0}^{+}$and $\bar{D}^{+}$be the operators restricted to the $(+)$-spinors corresponding to the $(+)$-spin representation. Then

(5.23) THEOREM.

$$
\begin{gathered}
\operatorname{Index}\left(\bar{D}_{0}^{+}\right)=\int_{X} \hat{A}(P)+\frac{\eta(0)-h}{2}-\sum_{0<\mu_{j}<1 / 2} \operatorname{dim}\left(E_{\mu_{j}}\right), \\
\operatorname{Index}\left(\bar{D}^{+}\right)=\int_{X} \hat{A}(P)+\frac{\eta(0)+h}{2}+\sum_{-1 / 2<\mu_{j}<0} \operatorname{dim}\left(E_{\mu_{j}}\right),
\end{gathered}
$$

where $\hat{A}$ is the Hirzebruch $\hat{A}$-polynomial of Pontrjagin forms $\left\{P_{i}\right\}, \eta(s)=$ $\sum_{\mu_{j} \neq 0}\left(\operatorname{sign} \mu_{j}\right)\left|\mu_{j}\right|^{-s}$ (the eta function), $h=\operatorname{dim} \operatorname{Ker}(\tilde{D})$, and $E_{\mu_{j}}$ is the eigenspace of $\tilde{D}$ with eigenvalue $\mu_{j}$.

When $\bar{D}$ is selfadjoint, i.e., $\left|\mu_{j}\right| \geqslant 1 / 2$, we have

$$
\operatorname{Index}\left(\bar{D}_{0}^{+}\right)=\operatorname{Index}\left(\bar{D}^{+}\right)=\int_{X} \hat{A}(p)+\frac{\eta(0)}{2} .
$$

Combined with the vanishing theorem (4.3), this gives

(5.24) THEOREM. Suppose that the scalar curvature $\kappa$ of $X=C_{0,1}(N) \cup M$ satisfies $\kappa \geqslant 0$ and $\kappa>0$ somewhere. Then

$$
\operatorname{Index}\left(\bar{D}^{+}\right)=0=\int_{X} \hat{A}(p)+\frac{\eta(0)}{2} .
$$

This gives a topological obstruction to the existence of the metrics with $\kappa \geqslant 0$ on singular spaces.

We conclude our paper by noting that all the results obtained for spinors immediately generalize to spinors with coefficients in a bundle $E$, i.e., to sections of the twisted bundle of spinors $S(X) \otimes E$, if the connection of $E$ is flat in a 
neighborhood of the singularity. The following vanishing theorem is an easy consequence. Set

$$
R_{0}(\sigma \otimes e)=\frac{1}{2} \sum_{j, k}\left(e_{j} e_{k} \sigma\right) \otimes R_{e_{j} e_{k}}^{E} e
$$

as in (1.11) and (1.12).

(5.28) Theorem. Suppose on $X=C_{0,1}(N) \cup M^{2 k}$ that $\kappa \geqslant 4\left\|R_{0}\right\|$ and $\kappa>4\left\|R_{0}\right\|$ somewhere. Then $\int_{X} \widehat{\mathrm{Ch}}(E) \cdot \hat{A}=0$, where $\widehat{\mathrm{Ch}}(E)$ is the reduced Chern character, $\widehat{\mathrm{Ch}}(E)=\mathrm{Ch}_{1}(E)+\mathrm{Ch}_{2}(E)+\cdots$.

We can also define the notation of enlargeability as in [17] (see (5.29)) and obtain a similar result for singular spaces.

(5.30) ThEOREM. Suppose in $X=C_{0,1}(N) \cup M^{2 k}$ that $M$ is of dimension $2 k$ and the interior of $M$ is enlargeable. Then there exists no metric, which is conical near the singularity at the cone tip, with scalar curvature such that $\kappa \geqslant 0$ and $\kappa>0$ on the interior of $M$.

We would like to make a final remark that these vanishing theorems and index theorems have interesting generalizations to pseudomanifolds. They are spaces which can be built up inductively by spaces with conical singularities (see $[7,8]$ ). To put this work in a better perspective, one could say that it serves as a first step toward some understanding of the piecewise linear geometries related to the Dirac operator, scalar curvatures, and eta-invariants.

ACKNOWLEDGMENTS. This work was done at SUNY-Stony Brook. The author wishes to express his gratitude to his advisor, Jeff Cheeger, for guidance and encouragement. He is grateful to Detlef Gromoll and Blaine Lawson for many helpful conversations and also wishes to thank the Stony Brook Mathematics Department for providing such an excellent environment.

1. Preliminaries. In this section we briefly recall some basic facts about spin manifolds and the Dirac operator. The general references are $[2,16,18,22,24]$.

An orientable manifold $X$ is called a spin manifold if its second Stiefel-Whitney class $W_{2}(X)$ is zero. Suppose $X$ is equipped with a Riemannian metric, and let $P_{\mathrm{SO}_{n}}(X)$ be the bundle of oriented orthonormal tangent frames. Let Spin ${ }_{n}$ denote the spin group, which is the universal 2-fold covering of $\mathrm{SO}_{n}$ for $n \geqslant 3$. A spin structure on $X$ is a principal $\operatorname{Spin}_{n}$-bundle $P_{\operatorname{Spin}_{n}}(X)$ together with a $\operatorname{Spin}_{n}$-equivariant map $\xi: P_{\operatorname{Spin}_{n}}(X) \rightarrow P_{\mathrm{SO}_{n}}(X)$ which commutes with the projection maps onto $X$. The condition $W_{2}(X)=0$ is equivalent to the existence of a spin structure. In fact, using Cech Cohomology, we can easily see that the toplogical obstruction cocycle to the globalization of the local 2-fold covering map

$$
\left.P_{\operatorname{Spin}_{n}}(X)\right|_{U} \cong \operatorname{Spin}_{n} \times\left. U \rightarrow P_{\mathrm{SO}_{n}}(X)\right|_{U} \cong \mathrm{SO}_{n} \times U,
$$

where $U$ is a small neighborhood, is exactly $W_{2}(X) \in H^{2}(X, Z)$.

Let $\mathrm{Cl}_{n}$ denote the Clifford algebra of $R^{n}$ with its standard inner product. In this paper we consider only the complexified Clifford algebra $\mathrm{Cl}_{n}=\mathrm{Cl}_{n} \otimes_{R} C$. The 
(complex) Spin representation of the Spin group $\operatorname{Spin}_{n}$ is, by definition, the restriction of the algebra representation $\rho$ of $\mathrm{Cl}_{n}$ to $\operatorname{Spin}_{n} \subset \mathrm{Cl}_{n}$. The Spin group $\operatorname{Spin}_{n}$ has only one irreducible representation if $n$ is odd and two irreducible representations $\Delta^{ \pm}$if $n$ is even. These two irreducible representations $\Delta^{ \pm}$of $\operatorname{Spin}_{2 k}$ come from the irreducible representation $\Delta$ of $\mathrm{Cl}_{2 k}$;

$$
\Delta: \mathrm{Cl}_{2 k} \stackrel{\cong}{\rightarrow} \operatorname{End}\left(C^{2^{k}}\right): \text { the group of endomorphisms of } C^{2^{k}} .
$$

When restricted to $\operatorname{Spin}_{2 k}, \Delta$ breaks into two irreducible representations $\Delta^{ \pm}$corresponding to the $( \pm)$-eigenspace of multiplication by the volume form $w=$ $i^{k} e_{1} e_{2}, \ldots, e_{2 k}$, where $\left\{e_{1}, \ldots, e_{2 k}\right\}$ is the orthonormal basis of $R^{2 k}$.

Suppose now that $X$ is a spin manifold of dimension $n$ and $P_{\operatorname{Spin}_{n}}(X) \rightarrow P_{\text {SO }_{n}}(X)$ is a spin structure on $X$. Then from the spin representation $\rho$ of $\operatorname{Spin}_{n}$, we can form the associated (complex) vector bundle

$$
S(X)=P_{\text {Spin }_{n}}(X) \times_{\rho} V,
$$

where $V$ is the representation space of $\rho$. This is called the bundle of (complex) spinors. If $n=2 k$, then (1.3) breaks into two pieces:

$$
\begin{aligned}
S(X) & =P_{\operatorname{Spin}_{n}}(X) \times_{\Delta} V \\
& =P_{\operatorname{Spin}_{n}}(X) \times_{\Delta+} V^{+} \oplus P_{\operatorname{Spin}_{n}}(X) \times_{\Delta-} V^{-}=S^{+}(X) \oplus S^{-}(X) .
\end{aligned}
$$

The sections of $S(X), \Gamma(S)$, are called spinors, and the sections of $S^{+}\left(S^{-}\right)$are called (+)-spinors ((-)-spinors). Let us denote the associated principle SO-bundle of $S(X)$ by $P_{S(X)}$. Then a local section $e=\left\{e_{1}, \ldots, e_{n}\right\}$ of $P_{\mathrm{SO}_{n}}(X)$ can be lifted up to $P_{\text {Spin }_{n}}(X)$ via (1.1) and then imbedded into $P_{S(X)}$ as a local section $\phi=\left\{\phi_{1}, \ldots, \phi_{N}\right\}$. This section $\phi$ is a local orthonormal basis of the bundle $S(X)$ and will be called the spinor basis.

Let $\mathrm{Cl}(X)$ denote the associated bundle of Clifford algebras. This is the bundle over $X$ whose fiber at each point $x$ is the (complex) Clifford algebra of the tangent space $T_{x}(X)$ with its given metric. This bundle carries a natural unitary connection $\nabla$, induced from the principal $\mathrm{SO}_{n}$-bundle, and characterized by the condition that $\nabla$ acts as a derivation on the algebra of sections $\Gamma(\mathrm{Cl}(X))$, i.e., $\nabla(\alpha \cdot \beta)=(\nabla \alpha) \cdot \beta$ $+\alpha \cdot(\nabla \beta)$ for all $\alpha, \beta \in \Gamma(\mathrm{Cl}(X))$, where $\cdot$ is Clifford multiplication.

We can easily see that $S(X)$ is a bundle of modules over $\mathrm{Cl}(X)$, i.e., there is a module multiplication

$$
\text { • : } \Gamma(\mathrm{Cl}) \times \Gamma(S) \rightarrow \Gamma(S) \text { defined by }(\alpha \cdot \phi)(x)=\rho(\alpha(x))(\phi(x))
$$

for all $\alpha \in \Gamma(\mathrm{Cl})$ and all $\phi \in \Gamma(S)$, where $\phi(x) \in V_{x}$ and $\rho(\alpha(x)) \in \operatorname{End}\left(V_{x}\right)$ (see (1.4)).

Lifting the Riemannian connection on $P_{\mathrm{SO}_{n}}(X)$ to $P_{\mathrm{Spin}_{n}}(X)$ via the Lie algebra isomorphism, we have that $\left(\operatorname{Spin}_{n}\right)_{*} \cong\left(\mathrm{SO}_{n}\right)_{*}$ determines an associated connection $\nabla^{s}$ on $S(X)$ whose action on the spinor basis $\phi=\left\{\phi_{1}, \ldots, \phi_{N}\right\}$ can be described as follows. Let $e=\left\{e_{1}, \ldots, e_{n}\right\}$ be a local section of $P_{\mathrm{SO}_{n}}(X)$ and $\nabla^{T}$ the Riemannian connection on the tangent bundle $T(X)$. Suppose that $\left\{\omega_{i j}\right\}$ are the 1-forms defined by

$$
\nabla^{T} e_{i}=\sum_{j=1}^{n} \omega_{i j} e_{j} \quad \text { for } i=1,2, \ldots, n
$$


Then

$$
\nabla^{s} \phi_{l}=\frac{1}{2} \sum_{i<j} \omega_{i j} e_{i} e_{j} \cdot \phi_{l} \quad \text { for } l=1,2, \ldots, N,
$$

where $\cdot$ is the module multiplication defined in (1.5).

It can also be shown that $\nabla^{s}$ acts as a derivation with respect to module multiplication, i.e.,

$$
\nabla^{s}(\alpha \cdot \phi)=(\nabla \alpha) \cdot \phi+\alpha \cdot\left(\nabla^{s} \phi\right)
$$

for all $\alpha \in \Gamma(\mathrm{Cl})$ and all $\phi \in \Gamma(S)$. From now on we drop the superscripts and simply use $\nabla$ to denote various connections if no ambiguity occurs.

The Dirac operator $D: C^{\infty}(S) \rightarrow C^{\infty}(S)$ is defined by

$$
D \phi=\sum_{i=1}^{n} e_{i} \cdot \nabla_{e_{i}} \phi,
$$

where $\left\{e_{1}, \ldots, e_{n}\right\}$ is a local orthonormal basis on $X$ and $\phi \in C^{\infty}(S)$. This is an invariantly defined first order elliptic differential operator with symbol $\sigma_{\xi}(D)=\xi$. for $\xi \in T^{*}(X)$. Notice that, in the case of an even-dimensional manifold, $D$ : $C^{\infty}\left(S^{ \pm}\right) \rightarrow C^{\infty}\left(S^{ \pm}\right)$, and we denote $\left.D\right|_{S^{ \pm}}$by $D^{ \pm}$.

Using the normal coordinate we can write

$$
D^{2} \phi=\sum_{i, j} e_{i} \cdot e_{j} \cdot \nabla_{e_{i}, e_{j}} \phi
$$

where $\nabla_{v, w}=\nabla_{v} \nabla_{w}-\nabla_{\nabla_{v}, w}$, and the symbol of $D^{2}$ is $\sigma_{\xi}\left(D^{2}\right)=-\|\xi\|^{2}$ for $\xi \in$ $T^{*}(X)$. Define

$$
\nabla^{2} \phi=\sum_{j=1}^{n} \nabla_{e_{j}, e_{j}} \phi \quad \text { for } \phi \in C^{\infty}(S) .
$$

Then the following relation holds pointwisely (cf. $[22,23])$.

(1.10) Theorem (Lichnerowicz - Bochner - Weitzenböck Formula). $D^{2} \phi=$ $-\nabla^{2} \phi+\frac{1}{4} \kappa \cdot \phi$, where $\kappa$ is the scalar curvature of $X$.

We can extend the above notions to more general classes of bundles. Suppose that $E$ is any hermitian vector bundle over $X$ with a unitary connection $\nabla^{E}$. Consider the bundle $S(X) \otimes E$ with the canonical tensor product connection $\nabla$. This is again a bundle of modules over $X$ satisfying (1.7). We can still define the (generalized) Dirac operator $D$ and $-\nabla^{2}$ by using this new connection $\nabla$, as in (1.8) and (1.9). Thus the corresponding formula to (1.10) is

$$
D^{2}=-\nabla^{2}+\frac{1}{4} \kappa+R_{0},
$$

where

$$
R_{0}(\phi \otimes \sigma)=\frac{1}{2} \sum_{i, j}\left(e_{i} \cdot e_{j} \cdot \phi\right) \otimes R_{e_{i}, e_{j}}^{E}(\sigma)
$$

for all $\phi \in S(X)$ and all $\sigma \in \Gamma(E)$, and $R^{E}$ denotes the curvature tensor of the connection $\nabla^{E}$. 
If $X$ is a closed manifold, then $D$ is selfadjoint, and $-\nabla^{2}=\nabla^{*} \cdot \nabla$ is selfadjoint and nonnegative. Thus (1.11) gives (see [16])

(1.13) THEOREM. If $\kappa>4\left\|R_{0}\right\|$, where \|\| is the operator norm, then $\operatorname{Ker}(D)=\{0\}$.

2. The separation of variables formula and eigenspinors of the Dirac operator on the cone. In this section we derive the separation of variables formula of the Dirac operator $D$ and $D^{2}$ on cones. We will also write down the eigenspinors of $D$ and $D^{2}$ explicitly and discuss the domains of closed extensions of them.

Recall that a cone on $N, C(N)$, is a space $(0, \infty) \times N$ with the metric of $d r \otimes d r+r^{2} \tilde{g}$, where $\tilde{g}$ is the metric on $N$. We assume that $N^{m}$ is a closed manifold of dimension $m$ and $C(N)$ is a spin manifold. Let $N$ be endowed with the induced spin structure from $C(N)$, i.e., the principal $\operatorname{Spin}_{m}$-bundle $P_{\operatorname{Spin}_{m}}(N)$ on $N$ is the reduction of $P_{\text {Spin }_{m+1}}(C(N))$ via the inclusion maps on $N^{m}$ :

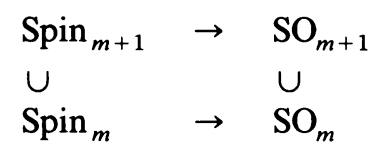

Let $\partial / \partial r$ denote the unit tangent vector to the radial geodesic $(0, \infty) \times\{x\}$ for some $x \in N$. Then the inclusion maps in (1.1) are just "adding $\partial / \partial r$ to the (oriented) orthonormal frames $\left(\tilde{e}_{1}, \ldots, \tilde{e}_{m}\right)$ on $N$ to form the (oriented) orthonormal frames $\left(\partial / \partial r, \tilde{e}_{1}, \ldots, \tilde{e}_{m}\right)$ on $C(N)$ ". This also defines the orientations on $C(N)$. By taking the induced spin representation of $\operatorname{Spin}_{m}$ from $\operatorname{Spin}_{m+1}$, the bundle of spinors $S(N)$ over $N$ can be canonically imbedded into the bundle of spinors $S(C(N))$ over $C(N)$. Let us denote everything intrinsic to $N$ by a tilde $\sim$ and the parallel translation along the radial geodesics by a bar -. Thus we can imbed a section $\tilde{\sigma}$ of $S(N)$ into $S(C(N))$ and then extend it to $\overline{\tilde{\sigma}}$ on $C(N)$ with the property that $\nabla_{\partial / \partial r} \overline{\tilde{\sigma}}=0$.

We now derive the separation of variables formula for a more general kind of metric on $(0, \infty) \times N: g=d r \otimes d r+h^{2}(r) \tilde{g}$ for some $h>0$.

(2.2) LemMA. Let $\left\{\tilde{e}_{i}, i=1,2, \ldots, m\right\}$ be a local orthonormal basis on $N$. Then $\left\{\partial / \partial r, E_{i}=\overline{\tilde{e}}_{i}, i=1,2, \ldots, m\right\}$ is a local orthonormal basis on $C(N)$, and

$$
\begin{aligned}
& \left\langle\nabla_{E_{i}} E_{j}, E_{k}\right\rangle=\frac{1}{h}\left\langle\nabla_{\tilde{e}_{i}} \tilde{e}_{j}, \tilde{e}_{k}\right\rangle_{\tilde{g}}, \\
& \left\langle\nabla_{E_{i}} E_{j}, \frac{\partial}{\partial r}\right\rangle=-\frac{h^{\prime}}{h} \delta_{i j} \quad \text { where } h^{\prime}=\frac{\partial}{\partial r} h, \\
& \left\langle\nabla_{E_{i}} \frac{\partial}{\partial r}, E_{j}\right\rangle=\frac{h^{\prime}}{h} \delta_{i j}, \quad \begin{array}{l}
\nabla \text { and }\langle,\rangle \text { are the Riemannian } \\
\text { connection and the inner product of } g,
\end{array} \\
& \left\langle\nabla_{E_{i}} \frac{\partial}{\partial r}, \frac{\partial}{\partial r}\right\rangle=0 .
\end{aligned}
$$

Proof. This follows in a straightforward way from the following formula for the Riemannian connection $\nabla$ (see [9]):

$$
\begin{aligned}
2\left\langle\nabla_{x} y, z\right\rangle= & x\langle y, z\rangle+y\langle x, z\rangle-z\langle x, y\rangle \\
& +\langle[x, y], z\rangle-\langle[x, z], y\rangle-\langle[y, z], x\rangle . \quad \text { Q.E.D. }
\end{aligned}
$$


Assume that $\left\{\sigma_{i}\right\}$ is a local spinor basis (see $\S 1$ ) of $S(N)$. Because $S(N)$ is induced from $S(C(N)),\left\{\bar{\sigma}_{i}\right\}$ is a local spinor basis of $S(C(N))$, and we have

(2.3) LEMMA. (1)

$$
\nabla_{\partial / \partial r} \bar{\phi}=0 \text { and } \nabla_{\partial / \partial r}(\partial / \partial r \cdot \bar{\phi})=0 \quad \forall \text { spinors } \phi \text { on } N,
$$

where $\cdot$ is module multiplication.

$$
\begin{gathered}
\nabla_{E_{i}} \bar{\sigma}_{j}=\frac{1}{2} \frac{h^{\prime}}{h} \frac{\partial}{\partial r} \cdot E_{i} \cdot \bar{\sigma}_{j}+\frac{1}{h} \overline{\tilde{\nabla}_{\tilde{e}_{i}} \sigma_{j}} \\
\nabla_{E_{i}}\left(\frac{\partial}{\partial r} \cdot \bar{\sigma}_{j}\right)=\frac{1}{2} \frac{h^{\prime}}{h} E_{i} \cdot \bar{\sigma}_{j}+\frac{1}{h} \frac{\partial}{\partial r} \cdot{\overline{\tilde{\nabla}_{\tilde{e}}}}_{\tilde{e}_{j}}
\end{gathered}
$$

Proof. (1) follows from the definition of the parallel translation and (1.7).

(2) We assume that $E_{0}=\partial / \partial r$. Then by (1.6),

$$
\begin{aligned}
\nabla_{E_{i}} \bar{\sigma}_{j} & =\frac{1}{2} \sum_{\substack{k<l=0 \\
k, l=0}}^{m} \omega_{k, l}\left(E_{i}\right) E_{k} E \bar{\sigma}_{j} \\
& =\frac{1}{2} \sum_{l=1}^{m} \omega_{0 l}\left(E_{i}\right) E_{0} E_{l} \bar{\sigma}_{j}+\frac{1}{2} \sum_{\substack{k=1 \\
k<l}}^{m} \omega_{k l}\left(E_{i}\right) E_{k} E_{l} \bar{\sigma}_{j} \\
& =\frac{1}{2} \frac{h^{\prime}}{h} \frac{\partial}{\partial r} E_{i} \bar{\sigma}_{j}+\frac{1}{2} \sum_{\substack{k=1 \\
k<l}}^{m} \tilde{\omega}_{k l}\left(E_{i}\right) E_{k} E_{l} \bar{\sigma}_{j} \\
& =\frac{1}{2} \frac{h^{\prime}}{h} \frac{\partial}{\partial r} E_{i} \overline{\boldsymbol{\sigma}}_{j}+\frac{1}{h} \bar{\nabla}_{\tilde{\boldsymbol{e}}_{i} \sigma_{j}}
\end{aligned}
$$

since $\omega_{k l}\left(\tilde{e}_{i}\right)=\tilde{\omega}_{k l}\left(e_{i}\right)$ for $k, l \geqslant 1$ by Lemma (2.2), where $\omega_{k l}$ and $\tilde{\omega}_{k l}$ are the connection 1-forms w.r.t. $\left\{E_{i}\right\}$ on $C(N)$ and $\left\{\tilde{e}_{i}\right\}$ on $N$, respectively, and $E_{i} \cdot \bar{\sigma}$ $=\overline{\tilde{e}_{i} \cdot \sigma}$. By using (1.7), (3) follows similarly. Q.E.D.

Notice that we use the same notation $\nabla$ for different connections whenever no ambiguity occurs.

(2.4) Proposition.

$$
\begin{gathered}
\nabla_{E_{i}} \bar{\phi}=\frac{1}{2} \frac{h^{\prime}}{h} \frac{\partial}{\partial r} E_{i} \bar{\phi}+\frac{1}{h} \overline{\tilde{\nabla}_{\tilde{e}_{i}} \phi} \\
\nabla_{E_{i}}\left(\frac{\partial}{\partial r} \cdot \bar{\phi}\right)=\frac{1}{2} \frac{h^{\prime}}{h} E_{i} \bar{\phi}+\frac{1}{h} \frac{\partial}{\partial r} \overline{\tilde{\nabla}_{\tilde{e}_{i} \phi}}
\end{gathered}
$$

for any spinor $\phi$ on $N^{m}$.

Proof. Write $\phi=\sum a_{i} \sigma_{i}$, where $\left\{\sigma_{i}\right\}$ is a spinor basis of $S(N)$ and apply (2.3). Q.E.D. 
The separation of variables formulas for $D$ and $D^{2}$ are the following:

(2.5) Proposition. Let

$$
\theta=f(r) \bar{\phi}+g(r) \frac{\partial}{\partial r} \bar{\omega},
$$

where $\bar{\phi}, \bar{\omega}$ are the parallel translations of the spinors $\phi, \omega$ on $N^{m}=\{1\} \times N^{m}$ to $C\left(N^{m}\right)$. Then

$$
\begin{aligned}
& D^{\theta}=\left(f^{\prime}+\frac{m}{2} \frac{h^{\prime}}{h} f\right) \frac{\partial}{\partial r} \bar{\phi}+\frac{1}{h} f \bar{D} \phi-\left(g^{\prime}+\frac{m}{2} \frac{h^{\prime}}{h} g\right) \bar{\omega}-\frac{1}{h} g \frac{\partial}{\partial r} \overline{D \omega} . \\
& D^{2} \theta=\left\{-f^{\prime \prime}-m\left(\frac{h^{\prime}}{h}\right) f^{\prime}-\left[\frac{m}{2}\left(\frac{h^{\prime}}{h}\right)^{\prime}+\left(\frac{m}{2} \frac{h^{\prime}}{h}\right)^{2}\right] f\right\} \bar{\phi} \\
& +\left\{\frac{h^{\prime}}{h^{2}}\right\} f \frac{\partial}{\partial r} \overline{D_{\phi}}+\left\{\frac{1}{h^{2}} f\right\} \overline{D^{2} \phi} \\
& +\left\{-g^{\prime \prime}-m\left(\frac{h^{\prime}}{h}\right) g^{\prime} e-\left[\frac{m}{2}\left(\frac{h^{\prime}}{h}\right)^{\prime}+\left(\frac{m}{2} \frac{h^{\prime}}{h}\right)^{2}\right] g\right\} \frac{\partial}{\partial r} \bar{\omega} \\
& +\left\{\frac{h^{\prime}}{h^{2}} g\right\} \bar{D} \omega+\left\{\frac{1}{h^{2}} g\right\} \frac{\partial}{\partial r} \overline{\tilde{D}^{2} \omega},
\end{aligned}
$$

where $\tilde{D}$ is the Dirac operator on $N^{m}$.

Proof. This is just a straightforward computation.

$$
\begin{aligned}
D \theta= & \frac{\partial}{\partial r} \nabla_{\partial / \partial r} \theta+\sum_{i=1}^{m} E_{i} \cdot \nabla_{E_{i}} \theta \\
= & \frac{\partial}{\partial r} \cdot\left\{f^{\prime}(r) \bar{\phi}+g^{\prime}(r) \frac{\partial}{\partial r} \bar{\omega}\right\} \\
& +\sum_{i=1}^{m} E_{i} \cdot\left\{f(r) \nabla_{E_{i}} \bar{\phi}+g(r) \nabla_{E_{i}} \frac{\partial}{\partial r} \bar{\omega}\right\} \\
= & f^{\prime}(r) \frac{\partial}{\partial r} \bar{\Phi}-g^{\prime}(r) \bar{\omega}+\frac{f(r)}{h} \bar{D} \phi+\frac{m}{2} \frac{h^{\prime}}{h} \frac{\partial}{\partial r} f(r) \bar{\phi} \\
& +\frac{g(r)}{h}\left(-\frac{\partial}{\partial r}\right) \overline{\tilde{D} \omega}-\frac{m}{2} \frac{h^{\prime}}{h} g(r) \bar{\omega} \\
= & {\left[f^{\prime}(r)+\frac{m}{2} \frac{h^{\prime}}{h} f(r)\right] \frac{\partial}{\partial r} \bar{\Phi}+\frac{f(r)}{h} \bar{D} \phi } \\
& -\left[g^{\prime}(r)+\frac{m}{2} \frac{h^{\prime}}{h} g(r)\right] \bar{\omega}-\frac{1}{h} g(r) \frac{\partial}{\partial r} \bar{D} \omega \\
D(D \theta)= & \frac{\partial}{\partial r} \nabla_{\partial / \partial r}(D \theta)+\sum_{i=1}^{m} E_{i} \cdot \nabla_{E_{i}}(D \theta) . \\
\nabla_{\partial / \partial r}(D \theta)= & {\left[f^{\prime}+\frac{m}{2}\left(\frac{h^{\prime}}{h}\right)^{\prime} f+\frac{m}{2} \frac{h^{\prime}}{h} f^{\prime}\right] \frac{\partial}{\partial r} \bar{\phi}-\frac{h^{\prime}}{h^{2}} f \tilde{D} \phi+\frac{1}{h} f^{\prime} \bar{D} \phi } \\
& -\left[g^{\prime \prime}+\frac{m}{2}\left(\frac{h^{\prime}}{h}\right)^{\prime} g+\frac{m}{2} \frac{h^{\prime}}{h} g^{\prime}\right] \bar{\omega}+\frac{h^{\prime}}{h^{2}} g \frac{\partial}{\partial r} \tilde{D} \omega-\frac{1}{h} g^{\prime} \frac{\partial}{\partial r} \overline{D \omega} .
\end{aligned}
$$




$$
\begin{aligned}
& \nabla_{E_{i}}(D)=\left(f^{\prime}+\frac{m}{2} \frac{h^{\prime}}{h} f\right)\left[\frac{1}{2} \frac{h^{\prime}}{h} E_{i} \bar{\phi}+\frac{1}{h} \frac{\partial}{\partial r} \overline{\tilde{\nabla}_{e_{i}} \phi}\right] \\
& +\left(\frac{1}{h} f\right)\left[\frac{1}{2} \frac{h^{\prime}}{h} \frac{\partial}{\partial r} E_{i} \tilde{D} \phi+\frac{1}{h} \overline{\tilde{\nabla}_{e_{i}}(\tilde{D} \phi)}\right] \\
& -\left(g^{\prime}+\frac{m}{2} \frac{h^{\prime}}{h} g\right)\left[\frac{1}{2} \frac{h^{\prime}}{h} \frac{\partial}{\partial r} E_{i} \bar{\omega}+\frac{1}{h} \overline{\tilde{\nabla}_{e_{i}} \omega}\right] \\
& -\left(\frac{1}{h} g\right)\left[\frac{1}{2} \frac{h^{\prime}}{h} E_{i} \overline{\tilde{D}_{\omega}}+\frac{1}{h} \frac{\partial}{\partial r} \overline{\tilde{\nabla}_{e_{i}}(\tilde{D} \omega)}\right] \text {. }
\end{aligned}
$$

Multiplying and collecting terms, we obtain the desired formula. Q.E.D.

Now we specialize to the metric $h(r)=r$. In order to treat spinors of the type $f(r) \bar{\phi}+g(r)(\partial / \partial r) \bar{\omega}$, it suffices to consider spinors of the type $f(r)(\bar{\phi} \pm(\partial / \partial r) \bar{\phi})$, since

$$
f \bar{\phi}=g \frac{\partial}{\partial r} \bar{\omega}=\frac{f}{2}\left(\bar{\phi}+\frac{\partial}{\partial r} \bar{\phi}\right)+\frac{f}{2}\left(\bar{\phi}-\frac{\partial}{\partial r} \phi\right)+\frac{g}{2}\left(\bar{\omega}+\frac{\partial}{\partial r} \bar{\omega}\right)-\frac{g}{2}\left(\bar{\omega}-\frac{\partial}{\partial r} \bar{\omega}\right) .
$$

Let $\phi$ be an eigenspinor of $\tilde{D}$ on $N^{m}$ with eigenvalue $\mu$, i.e., $\tilde{D} \phi=\mu \phi$, and suppose that $\theta=f(r)(\bar{\phi}+(\partial / \partial r) \bar{\phi})$ is an eigenspinor of $D^{2}$ with eigenvalue $\lambda^{2} \neq 0$. Then (2.5) becomes

$$
\begin{aligned}
D^{2} \theta & =\left\{-f^{\prime \prime}-\frac{m}{r} f^{\prime}+\left[\mu^{2}-\mu-\frac{m^{2}-2 m}{4}\right] \frac{1}{r^{2}} f\right\}\left(\bar{\phi}+\frac{\partial}{\partial r} \bar{\phi}\right) \\
& =\lambda^{2} f\left(\bar{\phi}+\frac{\partial}{\partial r} \bar{\phi}\right),
\end{aligned}
$$

hence

$$
+f^{\prime \prime}+\frac{m}{r} f^{\prime}+\left\{\lambda^{2}-\left[\mu^{2}-\mu-\frac{m^{2}-2 m}{4}\right] \frac{1}{r^{2}}\right\} f=0
$$

and the solutions are $f(r)=\gamma^{c} J_{ \pm \nu^{+}}(\lambda r)$, where

$$
c=(-m+1) / 2, \quad \nu^{+}=|2 \mu-1| / 2,
$$

and $J_{\nu}$ is the Bessel function of order $\nu$ (see [19]).

If $\theta=f(r)(\bar{\phi}-(\partial / \partial r) \bar{\phi})$ is an eigenspinor of eigenvalue $\lambda^{2} \neq 0$, then

$$
\begin{aligned}
D^{2} \theta & =\left\{-f^{\prime \prime}-\frac{m}{r} f^{\prime}+\left[\mu^{2}+\mu-\frac{m^{2}-2 m}{4}\right] \frac{1}{r^{2}} f\right\}\left(\bar{\phi}-\frac{\partial}{\partial r} \bar{\phi}\right) \\
& =\lambda^{2} f\left(\bar{\phi}-\frac{\partial}{\partial r} \bar{\phi}\right),
\end{aligned}
$$

hence

$$
+f^{\prime \prime}+\frac{m}{r} f^{\prime}+\left\{\lambda^{2}-\left[\mu^{2}+\mu-\frac{m^{2}-2 m}{4}\right] \frac{1}{r^{2}}\right\} f=0,
$$

and the solutions are $f(r)=r^{c} J_{ \pm \nu^{-}}(\lambda r)$, where $c=(-m+1) / 2, \nu^{-}=|2 \mu+1| / 2$, and $J_{\nu}$ is as before. 
Thus we have the following four types of eigenspinors of $D^{2}$ with eigenvalue $\lambda^{2} \neq 0$ :

$$
\begin{gathered}
r^{c} J_{\nu^{+}}(\lambda r)\left(\bar{\phi}+\frac{\partial}{\partial r} \bar{\phi}\right), \\
r^{c} J_{-\nu^{+}}(\lambda r)\left(\bar{\phi}+\frac{\partial}{\partial r} \bar{\phi}\right) \\
r^{c} J_{\nu^{-}}(\lambda r)\left(\bar{\phi}-\frac{\partial}{\partial r} \bar{\phi}\right) \quad \text { where } c=(1-m) / 2, \\
r^{c} J_{-\nu^{-}}(\lambda r)\left(\bar{\phi}-\frac{\partial}{\partial r} \bar{\phi}\right), \quad \nu^{ \pm}=\frac{|2 \mu+1|}{2} .
\end{gathered}
$$

The corresponding harmonic spinors, i.e., $\lambda^{2}=0$, are

$$
\begin{aligned}
& r^{c+\nu^{+}}\left(\bar{\phi}+\frac{\partial}{\partial r} \bar{\phi}\right), \\
& r^{c-\nu^{+}}\left(\bar{\phi}+\frac{\partial}{\partial r} \bar{\phi}\right), \\
& r^{c+\nu^{-}}\left(\bar{\phi}-\frac{\partial}{\partial r} \bar{\phi}\right), \\
& r^{c-\nu^{-}}\left(\bar{\phi}-\frac{\partial}{\partial r} \bar{\phi}\right) .
\end{aligned}
$$

Henceforth, we shall call (2.10), (2.12), (2.14), and (2.16) positive solutions, and (2.11), (2.13), (2.15), and (2.17) negative solutions. If $\nu^{ \pm}$is an integer, then we have to introduce logarithmic negative solutions. This can happen only when $\mu= \pm \frac{1}{2}$ and $\nu^{ \pm}=0$, and the negative solutions should be, instead of (2.11), (2.13),

$$
\begin{aligned}
& r^{c} Y_{0}(\lambda r)\left(\bar{\phi}+\frac{\partial}{\partial r} \bar{\phi}\right), \\
& r^{c} Y_{0}(\lambda r)\left(\bar{\rho}+\frac{\partial}{\partial r} \bar{\phi}\right),
\end{aligned}
$$

where $Y_{0}$ is the Bessel function of the second kind of order 0 .

Notice that

$$
Y_{0}(\lambda r) \sim \frac{2}{\pi} \log \frac{\lambda r}{2} \quad \text { as } r \rightarrow 0
$$

(see [21]). Similarly, (2.15) and (2.17) should be replaced by

$$
\begin{aligned}
& r^{c} \log r\left(\bar{\phi}+\frac{\partial}{\partial r} \bar{\phi}\right) \\
& r^{c} \log r\left(\bar{\phi}-\frac{\partial}{\partial r} \bar{\phi}\right)
\end{aligned}
$$


in this case. (2.14)-(2.17) can also be written in the following way:
(2.14)(a) $\quad r^{-m / 2+\mu}\left(\bar{\phi}+\frac{\partial}{\partial r} \bar{\phi}\right)$
if $\mu \geqslant \frac{1}{2}$,
(b) $\quad r^{-m / 2-\mu+1}\left(\bar{\phi}+\frac{\partial}{\partial r} \bar{\phi}\right)$
if $\mu<\frac{1}{2}$,
(2.15)(a) $\quad r^{-m / 2-\mu+1}\left(\bar{\phi}+\frac{\partial}{\partial r} \bar{\phi}\right)$
if $\mu>\frac{1}{2}$,
(b) $\quad r^{-m / 2+\mu}\left(\bar{\phi}+\frac{\partial}{\partial r} \bar{\phi}\right)$
if $\mu<\frac{1}{2}$,
(c) $\quad r^{c} \log r\left(\bar{\phi}+\frac{\partial}{\partial r} \bar{\phi}\right)$
if $\mu=\frac{1}{2}$,
(2.16)(a) $\quad r^{-m / 2+\mu+1}\left(\bar{\phi}-\frac{\partial}{\partial r} \bar{\phi}\right)$
if $\mu>-\frac{1}{2}$,
(b) $\quad r^{-m / 2-\mu}\left(\bar{\phi}-\frac{\partial}{\partial r} \bar{\phi}\right)$
if $\mu \leqslant-\frac{1}{2}$,
(2.17)(a) $\quad r^{-m / 2-\mu}\left(\bar{\phi}-\frac{\partial}{\partial r} \bar{\phi}\right)$
if $\mu>-\frac{1}{2}$,
(b) $r^{-m / 2+\mu+1}\left(\bar{\phi}-\frac{\partial}{\partial r} \bar{\phi}\right) \quad$ if $\mu<\frac{1}{2}$,
(c) $\quad r^{c} \log r\left(\bar{\phi}-\frac{\partial}{\partial r} \bar{\phi}\right)$
if $\mu=-\frac{1}{2}$.

Set $\nu_{1}=(2 \mu+1) / 2$ and $\nu_{2}=(2 \mu-1) / 2$.

Then the eigenspinors of $D^{2}$ can be classified as follows:
(1) $r^{c+\nu_{1}}\left(\bar{\phi}+\frac{\partial}{\partial r} \bar{\phi}\right)$
(I) $r^{c} J_{\nu_{1}}(\lambda r)\left(\bar{\phi}-\frac{\partial}{\partial r} \bar{\phi}\right)$
$=r^{-m / 2+\mu+1}\left(\frac{\bar{\phi}-\partial}{\partial r} \bar{\phi}\right)$,
(2) $r^{c-\nu_{1}}\left(\bar{\phi}-\frac{\partial}{\partial r} \bar{\phi}\right)$
(II) $r^{c} J_{-\nu_{1}}(\lambda r)\left(\bar{\phi}-\frac{\partial}{\partial r} \bar{\phi}\right)$,
$=r^{-m / 2-\mu}\left(\bar{\phi}-\frac{\partial}{\partial r} \bar{\phi}\right)$,
(2) $r^{\prime} \log r\left(\bar{\phi}-\frac{\partial}{\partial r} \bar{\phi}\right)$,
(3) $r^{c+\nu} 2\left(\bar{\phi}+\frac{\partial}{\partial r} \bar{\phi}\right)$
$=r^{-m / 2+\mu}\left(\bar{\phi}+\frac{\partial}{\partial r} \bar{\phi}\right)$,
(4) $r^{c-\nu_{2}}\left(\bar{\phi}+\frac{\partial}{\partial r} \bar{\phi}\right)$
(IV) $r^{c} J_{-\nu_{2}}(\lambda r)\left(\bar{\phi}+\frac{\partial}{\partial r} \bar{\phi}\right)$,
$=r^{-m / 2-\mu+1}\left(\bar{\phi}+\frac{\partial}{\partial r} \bar{\phi}\right)$,
(II) ${ }^{\prime} \quad r^{c} Y_{0}(\lambda r)\left(\bar{\phi}-\frac{\partial}{\partial r} \bar{\phi}\right)$,
(III) $r^{c} J_{\nu_{2}}(\lambda r)\left(\bar{\phi}+\frac{\partial}{\partial r} \bar{\phi}\right)$,
(4') $r^{c} \log r\left(\bar{\phi}+\frac{\partial}{\partial r} \bar{\phi}\right)$
$(\mathrm{IV})^{\prime} \quad r^{c} Y_{0}(\lambda r)\left(\bar{\phi}+\frac{\partial}{\partial r} \bar{\phi}\right)$ 
From (2.5) we have

$$
\begin{aligned}
& D\left[f\left(\bar{\phi}+\frac{\partial}{\partial r} \bar{\phi}\right)\right]=\left[-f^{\prime}-\frac{m / 2-\mu}{r} f\right]\left(\bar{\phi}-\frac{\partial}{\partial r} \bar{\phi}\right), \\
& D\left[f\left(\bar{\phi}-\frac{\partial}{\partial r} \bar{\phi}\right)\right]=\left[f^{\prime}+\frac{m / 2+\mu}{r} f\right]\left(\bar{\phi}+\frac{\partial}{\partial r} \bar{\phi}\right)
\end{aligned}
$$

Applying this to (2.19), we get

$$
\begin{aligned}
& D(1)=2 \nu_{1}(3), \quad D(2)=0, \quad D(2)^{\prime}=(3), \\
& D(3)=0, \quad D(4)=2 \nu_{2}(2), \quad D(4)^{\prime}=-(2) \text {. }
\end{aligned}
$$

Hence, (2), (3) are the only types of spinors in the kernel of $D$. Also, using the identities (see [21])

$$
J_{\nu}^{\prime}(z)-\frac{\nu}{z} J_{\nu}(z)=-J_{\nu+1}(z) \text { and } J_{\nu}^{\prime}(z)+\frac{\nu}{z} J_{\nu}(z)=J_{\nu-1}(z),
$$

we have

$$
\begin{array}{ll}
D(\mathrm{I})=\mathrm{l}(\mathrm{III}), & D(\mathrm{III})=\lambda(\mathrm{I}), \\
D(\mathrm{II})=-\lambda(\mathrm{IV}), & D(\mathrm{IV})=-\lambda(\mathrm{II}) .
\end{array}
$$

Thus we have the following two types of eigenspinors of $D$ with eigenvalue $\lambda \neq 0$ :

$$
\begin{gathered}
(\mathrm{I})+(\mathrm{III}): r^{c} J_{\nu_{1}}(\lambda r)\left(\bar{\phi}-\frac{\partial}{\partial r} \bar{\phi}\right)+r^{c} J_{\nu_{2}}(\lambda r)\left(\bar{\phi}+\frac{\partial}{\partial r} \bar{\phi}\right), \\
(\mathrm{II})-(\mathrm{IV}): r^{c} J_{-\nu_{1}}(\lambda r)\left(\bar{\phi}-\frac{\partial}{\partial r} \bar{\phi}\right)-r^{c} J_{-\nu_{2}}(\lambda r)\left(\bar{\phi}+\frac{\partial}{\partial r} \bar{\phi}\right) .
\end{gathered}
$$

Next we determine those eigenspinors of $D^{2}$ which are square-integrable when restricted to the finite cone $C_{0,1}(N)$. Let the domain of $D$ be

$$
\operatorname{dom}(D)=\left\{\phi \mid \phi \text { is } C^{\infty}, \phi \in L^{2}, \text { and } D \phi \in L^{2} \text { on the finite cone } C_{0,1}(N)\right\} .
$$

Since

$$
r^{a}\left(\bar{\phi} \pm \frac{\partial}{\partial r} \bar{\phi}\right) \in L^{2}\left(C_{0,1}(N)\right) \Leftrightarrow a>\frac{-1-m}{2},
$$

it follows, from (2.21), that in (2.19) we have
(1) (I) $\in \operatorname{dom}(D) \Leftrightarrow \mu>-1 / 2$,
(2) (II) $\in \operatorname{dom}(D) \Leftrightarrow \mu<1 / 2$,
(2)' (II)' $\notin \operatorname{dom}(D)$,
(3) (III) $\in \operatorname{dom}(D) \Leftrightarrow \mu>-1 / 2$,
(4) (IV) $\in \operatorname{dom}(D) \Leftrightarrow \mu<1 / 2$,
$(4)^{\prime} \quad(\mathrm{IV})^{\prime} \notin \operatorname{dom}(D)$.

$(2.24):(2.19)$

(2.25) Proposition. In the four types of eigenspinors of $D^{2}-(2.10)-(2.13)$ and (2.14)-(2.17) - the positive solutions are always in the domain of $D$. The negative solutions, corresponding to the small eigenvalues $\mu$ of $\tilde{D}$ such that $|\mu|<1 / 2$, are also in the domain of $D$. 
Proof. We need only examine (2.18) using (2.19), (2.24).

$$
\begin{gathered}
(2.15)(\mathrm{b}) \in \operatorname{dom}(D) \Leftrightarrow-\frac{1}{2}<\mu<\frac{1}{2}, \\
(2.17)(\mathrm{a}) \in \operatorname{dom}(D) \Leftrightarrow-\frac{1}{2}<\mu<\frac{1}{2} . \quad \text { Q.E.D. }
\end{gathered}
$$

We shall see that the negative solutions in the domain of $D$ are exactly the spinors which prevent $D$ from being selfadjoint.

Define $D_{0}$ to be the Dirac operator restricted to the spinors with compact support on $X^{m+1}=C_{0,1}\left(N^{m}\right) \cup M$. Let $\bar{D}_{0}$ and $\bar{D}$ denote the closures of these operators on $X$. Using the idea of the Friedrichs mollifier (see $[7,14,15])$, one can show that

$$
\bar{D}_{0}^{\prime}=\bar{D}, \quad \text { where }{ }^{*} \text { denotes adjoint. }
$$

Therefore $\bar{D}^{*}=\bar{D}$ is equivalent to $\bar{D} \subset \bar{D}^{*}$, i.e.,

$$
\langle\bar{D} \alpha, \beta\rangle=\langle\alpha, \bar{D} \beta\rangle \quad \text { for all } \alpha, \beta \in \operatorname{dom}(\bar{D}) \text {. }
$$

To show that this is not true in general, we first establish the following formula.

(2.28) Proposition (InTegration by Parts Formula). Let $Y^{m}$ be a compact spin manifold with boundary $\partial Y$, and let $\langle$,$\rangle denote the inner product on the space of L^{2}$ spinors. Suppose $\alpha$ and $\beta$ are smooth spinors. Then

$$
\langle D \alpha, \beta\rangle=\langle\alpha, D \beta\rangle-\int_{\partial Y}\langle\alpha, N \cdot \beta\rangle_{x},
$$

where $\langle,\rangle_{x}$ is the pointwise inner product on the fiber at $x$, and $N$ is the unit outer normal at the boundary.

$$
\begin{aligned}
& \text { Proof. }\langle D \alpha, \beta\rangle=\int_{Y}\langle D \alpha, \beta\rangle_{x} . \\
& \begin{aligned}
\langle D \alpha, \beta\rangle_{x} & =\sum_{i=1}^{m}\left\langle e_{i} \cdot \nabla_{e_{i}} \alpha, \beta\right\rangle \quad \begin{array}{l}
\text { where }\left\{e_{i}\right\} \text { is the orthonormal basis } \\
\text { of the tangent bundle, }
\end{array} \\
= & \sum_{i=1}^{m}\left\langle-\nabla_{e_{i}} \alpha, e_{i} \cdot \beta\right\rangle_{x} \\
& =\sum_{i=1}^{m}\left(e_{i}\left\langle-\alpha, e_{i} \cdot \beta\right\rangle_{x}-\left\langle-\alpha, \nabla_{e_{i}}\left(e_{i} \cdot \beta\right)\right\rangle_{x}\right) \\
& =-\sum_{i=1}^{m} e_{i}\left\langle-\alpha, e_{i} \cdot \beta\right\rangle_{x}+\sum_{i=1}^{m}\left\langle\alpha,\left(\nabla_{e_{i}} e_{i}\right) \cdot \beta\right\rangle_{x}+\langle\alpha, D \beta\rangle_{x} \\
& =-(\operatorname{div} V)_{x}+\langle\alpha, D \beta\rangle_{x},
\end{aligned}
\end{aligned}
$$

where $V$ is the vector field on $Y$ defined by

$$
(V, W)=\langle\alpha, W \cdot \beta\rangle \quad \forall \text { vector fields } W,
$$

and

$$
\operatorname{div} V=\sum_{i=1}^{m}\left(\nabla_{e_{i}} V, e_{i}\right)
$$


is the divergence of $V$. Hence,

$$
\begin{aligned}
\langle D \alpha, \beta\rangle & =\langle\alpha, D \beta\rangle-\int_{Y} \operatorname{div} V=\langle\alpha, D \beta\rangle-\int_{\partial Y}(V, N) \\
& =\langle\alpha, D \beta\rangle-\int_{\partial Y}\langle\alpha, N \cdot \beta\rangle . \quad \text { Q.E.D. }
\end{aligned}
$$

It follows that, on $X=C_{0,1}(N) \cup M$,

$$
\langle\bar{D} \alpha, \beta\rangle=\langle\alpha, \bar{D} \beta\rangle
$$

if $\alpha$ or $\beta$ has compact support. In order to show that $\bar{D}$ is selfadjoint, we have to prove that this is true for all $\alpha, \beta \in \operatorname{dom}(\bar{D})$, i.e., Stokes' theorem holds for $\bar{D}$. Unfortunately, this is not true in general because of the negative solutions in the domain of $\bar{D}$. The following example illustrates this situation.

(2.29) ExAmple. Let $X=C_{0,1}\left(N^{m}\right) \cup M$. Choose two negative solutions from (2.18), say,

$$
\begin{aligned}
& \theta_{1}=r^{-m / 2+\mu}\left(\bar{\phi}+\frac{\partial}{\partial r} \bar{\phi}\right), \\
& \theta_{2}=r^{-m / 2-\mu}\left(\bar{\phi}+\frac{\partial}{\partial r} \bar{\phi}\right),
\end{aligned}
$$

on $C_{0,1}(N)$, with $-1 / 2<\mu<1 / 2$. We can extend them to $X$ by multiplying by a cut-off function $f$, where $f(r) \equiv 1$ if $r<1-\varepsilon$ and $f(r) \equiv 0$ if $r \geqslant 1$ for some small number $\varepsilon$. Then $\theta_{1}, \theta_{2} \in \operatorname{dom}(\bar{D})$ on $X$. Let $X_{\varepsilon}=C_{\varepsilon, 1}(N) \cup M$. Then

$$
\begin{aligned}
\left\langle D \theta_{1}, \theta_{2}\right\rangle & =\int_{X}\left\langle D \theta_{1}, \theta_{2}\right\rangle=\lim _{\varepsilon \rightarrow 0} \int_{X_{\varepsilon}}\left\langle D \theta_{1}, \theta_{2}\right\rangle_{x} \\
& =\lim _{\varepsilon \rightarrow 0}\left(\int_{X_{\varepsilon}}\left\langle\theta_{1}, D \theta_{2}\right\rangle+\int_{\partial X_{\varepsilon}}\left\langle\theta_{1}, N \cdot \theta_{2}\right\rangle\right) \\
& =\left\langle\theta_{1}, D \theta_{2}\right\rangle+\lim _{\varepsilon \rightarrow 0} \int_{\partial X_{\varepsilon}}\left\langle\theta_{1}, N \cdot \theta_{2}\right\rangle \quad \text { by }(2.27) . \\
\lim _{\varepsilon \rightarrow 0} \int_{\partial X_{\varepsilon}}\left\langle\theta_{1}, N \cdot \theta_{2}\right\rangle & =\lim _{\varepsilon \rightarrow 0} \int_{\{\varepsilon\} \times N}\left\langle\theta_{1},\left(-\frac{\partial}{\partial r}\right) \cdot \theta_{2}\right\rangle \\
& =-\lim _{\varepsilon \rightarrow 0} \int_{\{\varepsilon\} \times N} 2\left\|\bar{\phi}^{2}\right\|^{-m} \cdot \varepsilon^{m} d V \neq 0,
\end{aligned}
$$

where $d V$ is the volume element on $\{1\} \times N^{m}$. Thus $\left\langle D \theta_{1}, \theta_{2}\right\rangle \neq\left\langle\theta_{1}, D \theta_{2}\right\rangle$ on $X$.

We can conclude that, in general, $\bar{D}_{0} \neq \bar{D}$ and the two selfadjoint extensions of $\left(D_{0}\right)^{2}, \Delta_{D}=\bar{D}_{0}^{*} \bar{D}_{0}$ and $\Delta_{N}=\bar{D} * \bar{D}$, are not the same.

(2.32) Remark. Since $\left\langle\bar{D}_{0} \alpha, \beta\right\rangle=\langle\alpha, \bar{D} \beta\rangle$ for all $\alpha \in \operatorname{dom}\left(\bar{D}_{0}\right)$ and $\beta \in$ $\operatorname{dom}(\bar{D})$, it can easily be shown, by using the same argument as in the previous example, that the negative solutions (2.11), (2.13), (2.15), (2.17) are not in the domain of $\bar{D}_{0}$.

3. The selfadjointness of the Dirac operator. In view of (2.29) and (2.32) it is natural to conjecture that $\bar{D}$ (or $\bar{D}_{0}$ ) is selfadjoint, provided there is no eigenvalue $\mu_{j}$ of $\tilde{D}$ on $N^{m}$ such that $\left|\mu_{j}\right|<1 / 2$. In fact, if we take for granted that the eigenvalues 
of $\bar{D} * \bar{D}$ on $X$ are discrete and the usual Fredholm theory holds for $\bar{D} * \bar{D}$ on $X$, which is established in $\S 5$, then it is easy to see that if $\left|\mu_{j}\right| \geqslant 1 / 2$, then $\langle\bar{D} \alpha, \beta\rangle=\langle\alpha, \bar{D} \beta\rangle$ holds for all eigenspinors $\alpha, \beta$ of $\bar{D} * \bar{D}$ and, hence, for all $\alpha, \beta \in \operatorname{dom}(\bar{D})$. Therefore the conjecture is proved.

In spite of this we prefer to show directly that the boundary term (2.31) goes to zero as the boundary approaches the cone tip, and, hence, (2.27) holds if $\left|\mu_{j}\right| \geqslant 1 / 2$. To this end we first obtain the following pointwise a priori estimate (compare [7]). The idea is to construct $D^{-1}$ via the separation of variables formula and then use Schwarz's inequality.

(3.1) Proposition. Suppose

$$
\alpha=f\left(\bar{\phi}+\frac{\partial}{\partial r} \bar{\phi}\right)+g\left(\bar{\phi}-\frac{\partial}{\partial r} \bar{\phi}\right)+\alpha^{+}+\alpha^{-} \in \operatorname{dom}(D)
$$

(see (2.23)), where $\phi$ is an eigenspinor of $D$ on $N$ with eigenvalue $\mu$. Denote the $L^{2}$-norm on $C_{0,1}(N)$ by \|\| and on $N$ by \|\|$_{N}$. Assume $\|\phi\|_{N}=1$. Then

$$
|f(r)| \leqslant \begin{cases}K\left\|\alpha^{+}\right\|(2 r)^{-m / 2+\mu}+\frac{1}{2}\left\|D \alpha^{+}\right\|\left|\frac{1-r^{-2 \mu+1}}{-2 \mu+1}\right|^{1 / 2} r^{-m / 2+\mu} & \text { if } \mu \neq \frac{1}{2}, \\ K\left\|\alpha^{+}\right\|(2 r)^{-m / 2+1 / 2}+\frac{1}{2}\left\|D \alpha^{+}\right\||\log r|^{1 / 2} r^{-m / 2+1 / 2} & \text { if } \mu=\frac{1}{2}\end{cases}
$$

where $K$ is a constant depending only on $m$. Moreover, if $\mu \leqslant-1 / 2$, then $D \alpha^{+} \neq 0$, and

$$
|f(r)| \leqslant \frac{1}{2}\left\|D \alpha^{+}\right\| r^{-m / 2+1 / 2} / \sqrt{-2 \mu+1} .
$$

Similarly,

$$
|g(r)| \leqslant \begin{cases}K\left\|\alpha^{-}\right\|(2 r)^{-m / 2-\mu}+\frac{1}{2}\left\|D \alpha^{-}\right\|\left|\frac{1-r^{2 \mu+1}}{2 \mu+1}\right|^{1 / 2} r^{-m / 2-\mu} & \text { if } \mu \neq \frac{1}{2}, \\ K\left\|\alpha^{-}\right\|(2 r)^{-m / 2+1 / 2}+\frac{1}{2}\left\|D \alpha^{-}\right\||\log r|^{1 / 2} r^{-m / 2+1 / 2} & \text { if } \mu=-\frac{1}{2} .\end{cases}
$$

If $\mu \geqslant \frac{1}{2}$ then $D \alpha^{-} \neq 0$, and

$$
|g(r)| \leqslant \frac{1}{2}\left\|D \alpha^{-}\right\| r^{-m / 2+1 / 2} / \sqrt{2 \mu+1} .
$$

Proof. Notice that, according to (2.19), (2.24), $D \alpha^{+}=0 \Rightarrow \mu>-1 / 2$, and $D \alpha^{-}=$ $0 \Rightarrow \mu<1 / 2$, since $\alpha \in \operatorname{dom}(D)$. The fact that $\alpha \in \operatorname{dom}(D)$ implies

$$
\int_{0}^{1}\left(f^{2}+g^{2}\right) r^{m} d r<\infty
$$

and, by (2.20),

$$
\int_{0}^{1}\left\{\left[f^{\prime}+\left(\frac{m}{2}-\mu\right) \frac{f}{r}\right]^{2}+\left[g^{\prime}+\left(\frac{m}{2}+\mu\right) \frac{g}{r}\right]^{2}\right\} r^{m} d r<\infty
$$

To obtain estimates for $f$ we first observe that

$$
\frac{\partial}{\partial r}\left(r^{m / 2-\mu} f\right)=r^{m / 2-\mu}\left[f^{\prime}+\left(\frac{m}{2}-\mu\right) \frac{f}{r}\right]
$$


and, hence,

$$
r^{m / 2-\mu} f=-\int_{r}^{a} s^{m / 2-\mu}\left[f^{\prime}+\left(\frac{m}{2}-\mu\right) \frac{f}{s}\right] d s+a^{m / 2-\mu} f(a) \quad \text { for any } a \in\left[\frac{1}{2}, 1\right] .
$$

By the Schwarz inequality with measure $r^{m} d r$,

$$
\begin{aligned}
\left|r^{m / 2-\mu} f(r)\right| \leqslant & \left(\int_{r}^{1} s^{-m-2 \mu} s^{m} d s\right)^{1 / 2}\left(\int_{r}^{1}\left[f^{\prime}+\left(\frac{m}{2}-\mu\right) \frac{f}{s}\right]^{2} s^{m} d s\right)^{1 / 2} \\
& +a^{m / 2-\mu}|f(a)| \\
= & a^{\mu}|f(a)|+\left|\frac{1-r^{-2 \mu+1}}{-2 \mu+1}\right|^{1 / 2}\left(\frac{1}{2}\left\|D \alpha^{+}\right\|\right), \text {if }-2 \mu+1 \neq 0
\end{aligned}
$$

and

$$
\left|r^{m / 2-\mu} f(r)\right| \leqslant a^{m / 2-\mu}|f(a)|+|\log r|^{1 / 2}\left(\frac{1}{2}\left\|D \alpha^{+}\right\|\right) \quad \text { if }-2 \mu+1=0 .
$$

Using the fact that there exist $a \in\left[\frac{1}{2}, 1\right]$ and a constant $K$ depending only on $m$ such that $|f(a)| \leqslant K \int_{0}^{1}|f|^{2} r^{m} d r$ (see [7]), we obtain the desired inequality.

Similarly, the inequality for $g$ follows.

Note that

$$
\begin{aligned}
\int_{0}^{r} r^{m / 2-\mu}\left(f^{\prime}+\left(\frac{m}{2}-\mu\right) \frac{f}{r}\right) d r & =\int_{0}^{r} \frac{\partial}{\partial r}\left(r^{m / 2-\mu} f\right) d r \\
& =r^{m / 2-\mu} f(r)-\lim _{r \rightarrow 0} r^{m / 2-\mu} f(r) .
\end{aligned}
$$

Since $\int_{0}^{1}|f|^{2} r^{m} d r<\infty$, we can find $r_{i} \rightarrow 0$ such that $\left|f^{2}\left(r_{i}\right) r_{i}^{m}\right|=o\left(1 / r_{i}\right)$, i.e., $\left|f\left(r_{i}\right)\right|=o\left(1 /(m+1) / r_{i}^{2}\right)$. (See [7].) Therefore, if $\mu<1 / 2$, the above limit exists and the integral converges. Moreover,

$$
\lim _{r \rightarrow 0} r^{m / 2-\mu} f(r)=\lim _{r_{i} \rightarrow 0} r_{i}^{m / 2-\mu} f\left(r_{i}\right)=\lim _{r_{i} \rightarrow 0} r_{i}^{m / 2-\mu} O\left(r_{i}^{-(m+1) / 2}\right)=0
$$

provided that $\mu \leqslant-1 / 2$.

Hence, we obtain that, if $\mu \leqslant-1 / 2$,

$$
\begin{aligned}
\left|r^{m / 2-\mu} f(r)\right| & =\left|\int_{0}^{r} \frac{\partial}{\partial r}\left(r^{m / 2-\mu} f\right)\right|=\left|\int_{0}^{r} s^{-m / 2-\mu}\left(f^{\prime}+\left(\frac{m}{2}-\mu\right) \frac{f}{s}\right) s^{m} d s\right| \\
& \leqslant\left|\int_{0}^{r}\left(s^{-m-2 \mu}\right) s^{m} d s\right|^{1 / 2}\left|\int_{0}^{r}\left[f^{\prime}+\left(\frac{m}{2}-\mu\right) \frac{f}{s}\right]^{2} s^{m} d s\right|^{1 / 2} \\
& =\left(\frac{r^{-2 \mu+1}}{-2 \mu+1}\right)^{1 / 2} \frac{1}{2}\left\|D \alpha^{+}\right\|,
\end{aligned}
$$

i.e.,

$$
|f(r)| \leqslant\left(r^{-m / 2+1 / 2} / \sqrt{-2 \mu+1}\right) \cdot \frac{1}{2}\left\|D \alpha^{+}\right\| .
$$

Similarly, we have

$$
|g(r)| \leqslant\left(r^{-m / 2+1 / 2} / \sqrt{-2 \mu+1}\right) \cdot \frac{1}{2}\left\|D \alpha^{-}\right\| \quad \text { if } \mu \geqslant \frac{1}{2} \text {. Q.E.D. }
$$


(3.2) THEOREM. Suppose $X^{m+1}=\overline{C_{0.1}\left(N^{m}\right)} \cup M$ is a compact space with a conical singularity. Let $D, \tilde{D}$ denote the Dirac operators on $X^{m+1}, N^{m}$, respectively, and let $\operatorname{dom}(D)=\left\{\phi \mid \phi \in C^{\infty} \cap L^{2}, D \phi \in L^{2}\right\}$. Then $\bar{D}$ is selfadjoint if and only if there is no eigenvalue of $\tilde{D}$ whose absolute value is strictly less than $1 / 2$.

Proof. To show that $\bar{D}$ is selfadjoint, it suffices to show that the boundary term in (2.30), (2.31) goes to zero. We need only check this for spinors of the form $f(\bar{\phi}+(\partial / \partial r) \bar{\phi})+g(\bar{\phi}-(\partial / \partial r) \bar{\phi})$, where $\phi$ is an eigenspinor of $\tilde{D}$ with eigenvalue $\mu$, and $\|\phi\|_{N}=1$. Let

$$
\begin{aligned}
& \theta_{1}=f_{1}\left(\bar{\phi}+\frac{\partial}{\partial r} \bar{\phi}\right)+g_{1}\left(\bar{\phi}-\frac{\partial}{\partial r} \bar{\phi}\right)=\theta_{1}^{+}+\theta_{1}^{-}, \\
& \theta_{2}=f_{2}\left(\bar{\phi}+\frac{\partial}{\partial r} \bar{\phi}\right)+g_{2}\left(\bar{\phi}-\frac{\partial}{\partial r} \bar{\phi}\right)=\theta_{2}^{+}+\theta_{2}^{-} .
\end{aligned}
$$

Assume that $\theta_{1}, \theta_{2} \in \operatorname{dom}(D)$ and $|\mu| \geqslant 1 / 2$. For the boundary term

$$
\int_{\{r\} \times N^{m}}\left\langle\theta_{1}, \frac{\partial}{\partial r} \cdot \theta_{2}\right\rangle=2\left(f_{2} g_{1}-f_{1} g_{2}\right) r^{m}
$$

(see (2.31)), we have two cases:

(i) $\mu \leqslant-1 / 2$. Then

$$
\begin{aligned}
& \left|f_{1}(r)\right| \leqslant c r^{-m / 2+1 / 2} \quad\left(\text { since } \mu \leqslant-1 / 2 \Rightarrow D\left(\theta_{1}^{+}\right) \neq 0\right), \\
& \left|g_{1}(r)\right| \leqslant c r^{-m / 2+1 / 2}, \\
& \left|f_{2}(r)\right| \leqslant c r^{-m / 2+1 / 2} \quad\left(\text { since } \mu \leqslant-1 / 2 \Rightarrow D\left(\theta_{2}^{+}\right) \neq 0\right), \\
& \left|g_{2}(r)\right| \leqslant c r^{-m / 2+1 / 2} .
\end{aligned}
$$

(ii) $\mu \geqslant 1 / 2$. Then

$$
\begin{aligned}
& \left|f_{1}(r)\right| \leqslant c r^{-m / 2+1 / 2}, \\
& \left|g_{1}(r)\right|<c r^{-m / 2+1 / 2} \quad\left(\text { since } \mu \geqslant 1 / 2 \Rightarrow D\left(\theta_{1}^{-}\right) \neq 0\right), \\
& \left|f_{2}(r)\right| \leqslant c r^{-m / 2+1 / 2}, \\
& \left|g_{2}(r)\right| \leqslant c r^{-m / 2+1 / 2} \quad\left(\text { since } \mu \geqslant 1 / 2 \Rightarrow D\left(\theta_{2}^{-}\right) \neq 0\right) .
\end{aligned}
$$

This shows $\left|f_{1} g_{2}-f_{2} g_{1}\right| r^{m} \rightarrow 0$ as $r \rightarrow 0$. Q.E.D.

(3.3) REMARKS. (1) This theorem says that $D_{0}$ is essentially selfadjoint (see [25]) if and only if $\left|\mu_{j}\right| \geqslant 1 / 2$ for all eigenvalues $\mu_{j}$ of $D$ on $N^{m}$.

(2) If the condition $\left|\mu_{j}\right| \geqslant 1 / 2$ is not satisfied, we can still obtain selfadjoint extensions of $D_{0}$ by imposing conditions on the domain of $D$, which correspond to the boundary conditions in the case of a manifold with boundary. Notice that in our case the boundary is crushed to a point: the singularity at the cone tip. We therefore call the conditions imposed on $\operatorname{dom}(D)$ to make it selfadjoint the ideal boundary conditions (see $[15,6,7])$. For example, suppose that $E=\bigoplus_{\left|\mu_{j}\right| \leqslant 1 / 2} E_{\mu_{j}}$, where $E_{\mu_{j}}$ is the eigenspace of $\tilde{D}$ with eigenvalue $\mu_{j}$. Let

$$
\left.\theta\right|_{E}=\sum f_{i}^{+}(r)\left(\bar{\phi}_{i}+\frac{\partial}{\partial r} \bar{\phi}_{i}\right)+f_{i}^{-}\left(\bar{\phi}_{i}-\frac{\partial}{\partial r} \bar{\phi}_{i}\right),
$$


where the $\phi_{i}$ are the eigenspinors of $D$ with eigenvalues $\mu_{j}$ such that $\left|\mu_{j}\right| \leqslant 1 / 2$, denote the projection of $\theta$ to the part consisting of eigenspinors in $E$ only. Define $D_{b}$ to be $D$ restricted to the space

$$
\left\{\theta \mid \theta \in L^{2} \text { and }\left.\theta\right|_{E} \text { satisfies }\left|f_{i}^{ \pm}\right|=o\left(r^{-m / 2}\right)\right\} .
$$

Then the proof of Theorem (3.2) tells us that the boundary term also goes to zero under this ideal boundary condition (3.4), i.e., $\bar{D}_{b} \subset \bar{D}_{b}^{*}$. But $\bar{D}_{0} \subset \bar{D}_{b}$ implies $\bar{D}_{b}^{*} \subset \bar{D}_{0}^{*}=\bar{D}$. It follows that $\bar{D}_{b}=D_{b}^{*}$ on the space (3.4).

(3) On a circle we have two spin structures. One is the trivial 2-fold covering; the other, the nontrivial spin structure induced from the disk. The first always gives a zero eigenvalue; and second has eigenvalues less than $1 / 2$ if the length is strictly less than $2 \pi$. This is the difficulty in generalizing our results to pseudomanifolds where 1-dimensional links always appear.

It is natural to ask for geometric conditions on $N^{m}$ which guarantee that $\left|\mu_{j}\right| \geqslant 1 / 2$. In fact, if the scalar curvature $\tilde{\kappa}$ of $N^{m}$ is positive and $k_{0}=\min \tilde{\kappa}$, then, using a slight modification of the Lichnerowicz-Bochner-Weitzenbock formula (1.10), we can show the following.

(3.5) LEMMA. $\left|\mu_{j}\right| \geqslant \frac{1}{2} \sqrt{m k_{0} /(m-1)}$, where $m=\operatorname{dim} N \geqslant 2$.

Proof. See [23 and 12].

Let $\kappa$ denote the scalar curvature of $C(N)$. It follows by straightforward computation (see (A.8) in the Appendix to $\$ 4)$ that $\kappa=1 / r^{2}[\kappa-m(m-1)]$. Therefore, if $\kappa \geqslant 0, \tilde{\kappa} \geqslant m(m-1)$. It follows from (3.5) that $|\mu| \geqslant m / 2$, and, hence,

(3.6) THEOREM. Let $X^{m+1}=C_{0,1}\left(N^{m}\right) \cup M$ and $m \geqslant 2$. Then $\bar{D}$ is selfadjoint if the scalar curvature of $C_{0,1}\left(N^{m}\right)$ is nonnegative.

4. Vanishing theorems. We prove vanishing theorems for singular spaces analogous to that proved by Lichnerowicz [23] for smooth manifolds. We begin with some general facts for arbitrary (possibly incomplete) manifolds.

(4.1) Lemma. Let $Y$ be an arbitrary spin manifold. Assume that the scalar curvature $\kappa$ of $Y$ satisfies $\kappa \geqslant-k$ for some positive constant $k$. Then we have

$$
\|D \theta\|^{2} \geqslant\|\nabla \theta\|^{2}-\frac{1}{4} \int_{Y} \kappa\|\theta\|_{x}^{2} \text { for all } \theta \in \operatorname{dom}\left(\bar{D}_{0}\right),
$$

where $\nabla$ is the connection, \|\| denotes the global $L^{2}$-norm on $Y$, and \|\|$_{x}$ the pointwise norm at $x$.

PROOF. Integrating the Lichnerowicz formula (1.10) $D^{2} \phi=-\nabla^{2} \phi+\frac{1}{4} \kappa \phi$ for smooth $\phi$ with compact support over $Y$, we immediately see that

$$
\begin{aligned}
\|D \theta\|^{2} & =\|\nabla \theta\|^{2}+\frac{1}{4} \int_{Y} \kappa\|\theta\|_{x}^{2} \\
& \geqslant\|\nabla \theta\|^{2}-\frac{1}{4} k\|\theta\| \quad \text { for all } \theta \in \operatorname{dom}\left(D_{0}\right) .
\end{aligned}
$$


Now let $\theta \in \operatorname{dom}\left(\bar{D}_{0}\right)$. By definition we can find $\theta_{i} \in \operatorname{dom}\left(D_{0}\right)$ such that $\theta_{i} \rightarrow \theta$ and $D \theta_{i} \rightarrow D$ as $i \rightarrow \infty$, in the $L^{2}$ sense. Since

$$
\left\|D \theta_{i}\right\|^{2}=\left\|\nabla \theta_{i}\right\|^{2}+\frac{1}{4} \int_{y} \kappa\left\|\theta_{i}\right\|_{x}^{2} \geqslant\left\|\nabla \theta_{i}\right\|^{2}-\frac{1}{4} k\left\|\theta_{i}\right\|,
$$

if we define

$$
\|\theta\|_{D}=\|D \theta\|+\left(\frac{1}{4} k+1\right)\|\theta\| \quad \text { and } \quad\|\theta\|_{\nabla}=\|\nabla \theta\|+\|\theta\|,
$$

then clearly \|\|$_{D} \geqslant\|\|_{\nabla}$. Therefore $\theta_{i}$ is a Cauchy sequence in the norm \|\|$_{\nabla}$ and, hence, converges to $\theta^{\prime}$ for some $\theta^{\prime}$. But \|\|$_{\nabla} \geqslant\|\|$, so we must have $\theta^{\prime}=\theta$, i.e, $\nabla \theta_{i} \rightarrow \nabla \theta$ in the $L^{2}$-norm \|\| . Thus,

$$
\begin{aligned}
\|D \theta\|^{2} & =\lim _{i \rightarrow \infty}\left\|D \theta_{i}\right\|^{2}=\lim _{i \rightarrow \infty}\left(\left\|\nabla \theta_{i}\right\|^{2}+\frac{1}{4} \int_{Y} \kappa\left\|\theta_{i}\right\|_{x}^{2}\right) \\
& =\left(\lim _{i \rightarrow \infty}\left\|\nabla \theta_{i}\right\|^{2}\right)+\lim _{i \rightarrow \infty} \frac{1}{4} \int_{Y} \kappa\left\|\theta_{i}\right\|_{x}^{2} \\
& =\|\nabla \theta\|^{2}+\frac{1}{4} \lim _{i \rightarrow \infty} \int_{Y} \kappa\left\|\theta_{i}\right\|_{x}^{2} .
\end{aligned}
$$

An application of Fatou's lemma yields

$$
\|D \theta\|^{2} \geqslant\|\nabla \theta\|^{2}+\frac{1}{4} \int_{Y} \kappa\|\theta\|_{x}^{2} \text {. Q.E.D. }
$$

(4.2) THEOREM. Let $Y$ be an arbitrary spin manifold with scalar curvature $\kappa$. Assume that $\kappa \geqslant 0$ and $\kappa>0$ somewhere. Then $\operatorname{Ker}\left(\bar{D}_{0}\right)=\{0\}$.

Proof. Let $\theta \in \operatorname{Ker}\left(\bar{D}_{0}\right)$. Then (4.1) gives

$$
0=\|D \theta\|^{2} \geqslant\|\nabla \theta\|^{2}+\frac{1}{4} \int_{Y} \kappa\|\theta\|^{2} .
$$

Since $\kappa>0$ on an open subset $U$, we have $\theta \equiv 0$ on $U$ and $\nabla \theta \equiv 0$ on $Y$. This implies $\theta \equiv 0$ on $Y$. Q.E.D.

Now let $X^{m+1}=C_{0,1}\left(N^{m}\right) \cup M$. Then $\kappa \geqslant 0$ guarantees that $\bar{D}$ is selfadjoint, and $\bar{D}_{0}=\bar{D}$ by (3.6). Thus, we have

(4.3) THEOREM. Suppose that on $X^{m+1}(m \geqslant 2), \kappa \geqslant 0$ and $\kappa>0$ somewhere. Then $\operatorname{Ker}\left(\bar{D}^{*} \bar{D}\right)=\operatorname{Ker}(\bar{D})=\{0\}$, i.e., there exists no square-integrable harmonic spinors on $X$.

(4.4) Remarks. (1) This theorem can also be proved directly by showing $\left\langle-\nabla^{2} \theta, \theta\right\rangle \geqslant 0$ for all $\theta$ in $\operatorname{Ker}(\bar{D})$ under the condition $\kappa \geqslant 0$.

(2) The above theorem can be construed as giving necessary conditions for a manifold with boundary to admit a metric with nonnegative scalar curvature for which the metric near the boundary is conical, i.e., like the exterior of a cone. In the Appendix to this section we give a geneal discussion of manifolds with boundary. 
(4.2) and (4.3) can easily be generalized to the generalized Dirac operator on the "twisted" bundle of spinors $S(X) \otimes E$, i.e., on spinors with coefficients in the bundle $E$ (see $\S 1$ ), if the connection on $E$ is flat in a neighborhood of the singularity at the cone tip $\{P\}$. For this situation we need only replace a spinor by an $n$-tuple of spinors $(n=\operatorname{dim} E)$ in our previous calculation while everything else stays the same. Under these assumptions we thus have (see (1.12), (1.13))

(4.5) TheOREM. Suppose that on $X^{m+1}(m \geqslant 2), \kappa>4\left\|R_{0}\right\|$. Then $\bar{D}$ is selfadjoint and $\operatorname{Ker}(\bar{D})=\{0\}$ for the generalized Dirac operator $\bar{D}$.

Proof. Since $E$ is flat near the cone tip, we can deduce from (1.12) that $R_{0} \geqslant-k$ for some positive $k$. Therefore the same arguments as in the proofs of (4.1), (4.2) give us the desired result.

Appendix to $\S 4$. Deformations of the metric near a boundary and positive scalar curvatures.

1. Introduction. On a manifold with boundary, a differential operator which is selfadjoint on a closed manifold is no longer selfadjoint without a suitable boundary condition. In certain situations we do not even know how to choose boundary conditions which make the problem meaningful. One way of avoiding this difficulty is to trivialize the geometry near the boundary and treat the space as part of an ambient space formed by attaching a cylinder (or a cone) to the boundary. We then study the operator on the ambient space in which no boundary is present. This is a basic point in the proof of the geometric index theorem for manifolds with boundary (see [3]).

In this Appendix we study two types of deformations of the metric near the boundary and their influence on the scalar curvature. We then obtain sufficient conditions under which the scalar curvature remains positive after the deformation. It follows from (A.8) that if the cylinder $N \times R$ has scalar curvature $\kappa>0$, then we can deform the metric slowly to a cone and keep the scalar curvature positive. Conversely, if the cone has $\kappa>0$, then, using the bending technique of Gromov and Lawson (see below and [17]), we can open up the cone tip to form a cylinder and maintain positive scalar curvature. Therefore, under this circumstance, attaching a cone to the boundary is equivalent to attaching a cylinder. But our vanishing theorem, when construed as for manifolds with boundary, emphasizes the case that $\kappa \equiv 0$ on the conical neighborhood of the boundary, for which no such deformation can be applied.

2. The bending technique. Let $M^{m+1}$ be a manifold with boundary $N^{m}$. We can find an $\varepsilon$-neighborhood of $N$ that is diffeomorphic to $[0, \varepsilon] \times N=N_{\varepsilon}$. Let $r$ denote the geodesic distance to the boundary, and let $g(r, x)$ be the metric on the hypersurface $\{r\} \times N$ at distance $r$ to the boundary. It is easy to see that the metric on this tubular neighborhood can be written as $d r \otimes d r+g(r, x)$. 
The first method of deformation is an adaptation of the "bending technique" due to Gromov and Lawson [17] for the connected sum of two manifolds. Define a hypersurface $M^{\prime}$ in $M \times R$ with the product metric by

$$
\begin{aligned}
M^{\prime}= & \{(y, t) \mid(y, t) \in M \times R \text { and }(\|y\|, t) \in \Gamma\}, \\
& \text { where }\|y\| \text { denotes the geodesic distance from } y \\
& \text { to the boundary } N, \text { i.e., }\|y\|=r, \text { and } \Gamma \text { is the } \\
& \text { curve in the }(r, t) \text { plane as described in the figure. }
\end{aligned}
$$

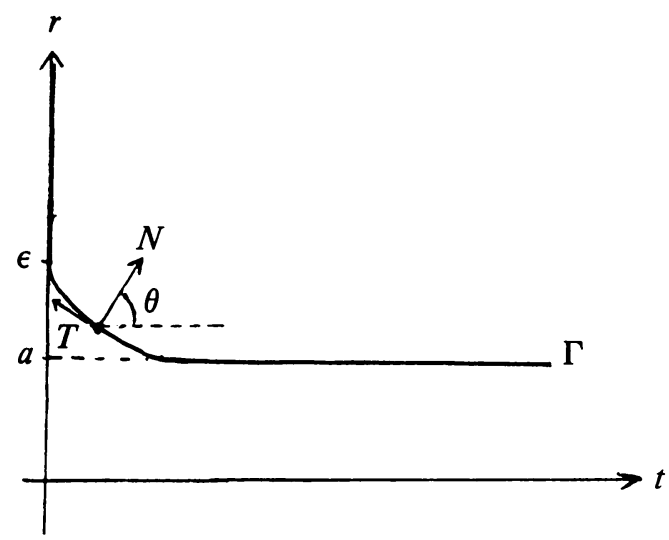

$\Gamma$ starts from the $r$-axis and finishes with a horizontal line to the $t$-axis at $r=a$.

Notice that only the part $N_{\varepsilon}$ is bent. The induced metric on $M^{\prime}$ from $M \times R$ extends the metric on $M \backslash N_{\varepsilon}$ smoothly and ends with the product metric on $(\{a\} \times N) \times N$. To study the change of the curvature under this bending, we begin with the following observations. (See also [17].)

(i) In $N_{\varepsilon}$ let $\partial / \partial r$ denote the tangent vector to the geodesic $[0, \varepsilon] \times\{x\}=l$. Let $N$ be the unit normal to $M^{\prime}$, and let $\bar{\nabla}$ be the connection on $M \times R$. Define the principal curvatures to be the eigenvalues of the operator $\bar{S}(X)=\bar{\nabla}_{x} N$. Then $\gamma_{l}=(l \times R) \cap M^{\prime}$ is a principal curve on $M^{\prime}$, i.e., the tangent vector $T$ to $\gamma_{l}$ is a principal direction of the second fundamental form $\bar{S}$, and the associated principal curvature at a point corresponding to $(r, t) \in \Gamma$ is exactly $-k$, where $k$ is the curvature of $\Gamma$ at that point.

(ii) Let $\theta$ be the angle between $N$ and the $t$-direction, and let $\left\{e_{1}, \ldots, e_{m}\right\}$ be the orthonormal basis of principal vectors for the operator $S(X)=\nabla_{x}(\partial / \partial r)$ on the hypersurface $\{r\} \times N$ with principal curvatures $\lambda_{i}, i=1, \ldots, m$. Then $\left\{e_{1}, \ldots, e_{m}\right\}$ are the principal vectors for $\bar{S}$ on $M^{\prime}$ with principal curvatures $\bar{\lambda}_{i}=\lambda_{i} \sin \theta$, since

$$
N=(\sin \theta) \frac{\partial}{\partial r}+(\cos \theta) \frac{\partial}{\partial t}
$$

and

$$
\begin{aligned}
\bar{\nabla}_{e_{i}} N & =\bar{\nabla}_{e_{i}}\left((\sin \theta) \frac{\partial}{\partial r}+(\cos \theta) \frac{\partial}{\partial t}\right)=(\sin \theta) \bar{\nabla}_{e_{i}} \frac{\partial}{\partial r} \\
& =(\sin \theta) \nabla_{e_{i}} \frac{\partial}{\partial r}=\left(\lambda_{i} \sin \theta\right) e_{i} .
\end{aligned}
$$


(iii) Let $K_{i j}, \bar{K}_{i j}$, and $K_{i j}^{\prime}$ denote the sectional curvature of $M, M \times R$, and $M^{\prime}$, respectively, corresponding to the plane $e_{i} \wedge e_{j}$. Set $e_{0}=T=\cos \theta(\partial / \partial r)+$ $\sin \theta(\partial / \partial t)$. By the Gauss curvature equation,

$$
K_{i j}^{\prime}=\bar{K}_{i j}+\bar{\lambda}_{i} \bar{\lambda}_{j} \quad \text { where } \bar{\lambda}_{0}=-k \text { and } \bar{\lambda}_{j}=\lambda_{j} \sin \theta \text { for } j \geqslant 1 .
$$

Since $M \times R$ has the product metric, one sees that

$$
\bar{K}_{0 j}=K_{\partial / \partial r, j} \cos ^{2} \theta, \quad \bar{K}_{i j}=K_{i j} \text { for } i, j \geqslant 1 .
$$

Hence,

$$
\begin{gathered}
K_{0 j}^{\prime}=K_{\partial / \partial r, j} \cos ^{2} \theta-k \lambda_{j} \sin \theta \\
K_{i j}^{\prime}=K_{i j}+\lambda_{i} \lambda_{j} \sin ^{2} \theta \text { for } i, j \geqslant 1
\end{gathered}
$$

It follows that the scalar curvature $\kappa^{\prime}$ of $M^{\prime}$ is given by

$$
\begin{aligned}
\kappa^{\prime}= & \sum_{\substack{i, j=1 \\
i \neq j}}^{m} K_{i j}^{\prime}=\sum_{\substack{i, j=1 \\
i \neq j}}^{m}\left(K_{i j}+\lambda_{i} \lambda_{j} \sin ^{2} \theta\right) \\
& +2 \sum_{j=1}^{m} K_{\partial / \partial r, j} \cos ^{2} \theta-2 k \sum_{j=1}^{m} \lambda_{j} \sin \theta \\
= & \kappa-2 \operatorname{Ric}\left(\frac{\partial}{\partial r}, \frac{\partial}{\partial r}\right)+\sum_{\substack{i, j=1 \\
i \neq j}}^{m} \lambda_{i} \lambda_{j} \sin ^{2} \theta \\
& +2 \operatorname{Ric}\left(\frac{\partial}{\partial r}, \frac{\partial}{\partial r}\right) \cos ^{2} \theta-2 k \sum_{\substack{j=1 \\
j}}^{m} \lambda_{j} \sin \theta \\
= & \kappa-2 \operatorname{Ric}\left(\frac{\partial}{\partial r}, \frac{\partial}{\partial r}\right) \sin ^{2} \theta+\sum_{\substack{i, j=1 \\
i \neq j}}^{m} \lambda_{i} \lambda_{j} \sin ^{2} \theta \\
& -2 k \sum_{j=1}^{m} \lambda_{j} \sin \theta,
\end{aligned}
$$

where $\kappa$ and Ric denote the scalar and Ricci curvatures of $M$, respectively.

Let us denote the scalar curvature of the hypersurfaces $\{r\} \times N$ in $M$ by $\kappa_{r}$. Using the formula

$$
\kappa_{r}=\kappa-2 \operatorname{Ric}\left(\frac{\partial}{\partial r}, \frac{\partial}{\partial r}\right)+\sum_{\substack{i, j=1 \\ i \neq j}}^{m} \lambda_{i} \lambda_{j}
$$

we deduce

$$
\begin{aligned}
\kappa^{\prime}= & \kappa+\left(\kappa_{r}-\kappa-\sum \lambda_{i} \lambda_{j}\right) \sin ^{2} \theta+\sum \lambda_{i} \lambda_{j} \sin ^{2} \theta \\
& -2 k\left(\sum \lambda_{j}\right) \sin \theta \\
= & \kappa \cos ^{2} \theta+\kappa_{r} \sin ^{2} \theta-2 k(\operatorname{Tr} S) \sin \theta,
\end{aligned}
$$

where $\operatorname{Tr} S$ is the trace of $S(X)=\nabla_{X}(\partial / \partial r)$. 
It follows that

(A.2) THEOREM. Let $M^{m+1}$ be a manifold with boundary $N^{m}$. Suppose

(i) $\kappa \geqslant 0$,

(ii) $\operatorname{Tr} S \leqslant 0$, i.e., the trace of the second fundamental form points inward,

(iii) $\kappa_{r} \geqslant 0$ for $r \in[0, \varepsilon]$.

Then in this tubular neighborhood $[0, \varepsilon] \times N$ the metric can be deformed to a complete metric, which ends with the product metric $N \times R$ near infinity, with scalar curvature $\kappa^{\prime} \geqslant 0$. Moreover, if $\kappa>0, \operatorname{Tr} S \leqslant 0$, and $\kappa_{r}>0$, then $\kappa^{\prime}>0$.

3. The deformation technique. Let $\bar{g}=d r^{2}+g(r, x)$ be the metric near the boundary $N^{m}$. We shall consider the deformation of the type $\phi^{2}(r) d r^{2}+$ $f^{2}(r) g(r, x)$. From now on we assume that $\operatorname{dim} N=m \geqslant 2$.

First let $g=d r^{2}+f^{2}(r) g(r, x)$, and let $\nabla$ denote its connection. Let $\bar{\nabla}$ denote the connection of the original metric $\bar{g}, \bar{g}=\langle,\rangle_{0}$, and $g=\langle$,$\rangle . Now if \left\{\partial / \partial r, \bar{e}_{i}\right\}$ is an orthonormal basis of $\bar{g}$, then $\left\{\partial / \partial r, e_{i}=\bar{e}_{i} / f\right\}$ is an orthonormal basis of $g$. A straightforward computation gives

(A.3) LEMMA.

$$
\begin{gathered}
\nabla_{\partial / \partial r} e_{j}=\frac{1}{f} \bar{\nabla}_{\partial / \partial r} \bar{e}_{j}=\frac{f^{\prime}}{f} e_{j}+\bar{\nabla}_{\partial / \partial r} e_{j} . \\
\nabla_{e_{i}} e_{j}=-\frac{f^{\prime}}{f} \delta_{i j} \frac{\partial}{\partial r}+\left(f^{2}-1\right)\left\langle\bar{\nabla}_{e_{i}} e_{j}, \frac{\partial}{\partial r}\right\rangle \frac{\partial}{\partial r}+\bar{\nabla}_{e_{i}} e_{j} \\
=-\frac{f^{\prime}}{f} \delta_{i j} \frac{\partial}{\partial r}-\left(1-\frac{1}{f^{2}}\right)\left\langle\bar{S}\left(e_{i}\right), e_{j}\right\rangle \frac{\partial}{\partial r}+\bar{\nabla}_{e_{i}} e_{j},
\end{gathered}
$$

where $\bar{S}\left(e_{i}\right)=\bar{\nabla}_{e_{i}}(\partial / \partial r)$.

$$
\nabla_{e_{i}} \frac{\partial}{\partial r}=\frac{f^{\prime}}{f} e_{i}+\bar{\nabla}_{e_{i}} \frac{\partial}{\partial r}
$$
have

Set $A\left(\bar{e}_{i}\right)=\left\langle\bar{\nabla}_{\bar{e}_{i}}(\partial / \partial r), \bar{e}_{i}\right\rangle_{0}$. Then $A\left(\bar{e}_{i}\right)=\left\langle\bar{S}\left(\bar{e}_{i}\right), \bar{e}_{i}\right\rangle_{0}=\left\langle\bar{S}\left(e_{i}\right), e_{i}\right\rangle$, and we (A.4) LEMMA.

$$
\begin{aligned}
&\left\langle\nabla_{e_{i}} \nabla_{e_{j}} e_{j}, e_{i}\right\rangle=-\left(\frac{f^{\prime}}{f}\right)^{2}-\frac{f^{\prime}}{f}\left[A\left(\bar{e}_{i}\right)+A\left(\bar{e}_{j}\right)\right] \\
&+\left(\frac{1}{f^{2}}-1\right) A\left(\bar{e}_{i}\right) A\left(\bar{e}_{j}\right)+\left\langle\bar{\nabla}_{e_{i}} \bar{\nabla}_{e_{j}} e_{j}, e_{i}\right\rangle . \\
&\left\langle\nabla_{e_{j}} \nabla_{e_{i}} e_{j}, e_{i}\right\rangle=\left(\frac{1}{f^{2}}-1\right)\left(\left\langle\bar{S}\left(e_{i}\right), e_{j}\right\rangle\right)^{2}+\left\langle\bar{\nabla}_{e_{j}} \bar{\nabla}_{e_{i}} e_{j}, e_{i}\right\rangle . \\
&\left\langle\nabla_{\left[e_{i}, e_{j}\right]} e_{j}, e_{i}\right\rangle=\left\langle\bar{\nabla}_{\left[e_{i}, e_{j}\right]} e_{j}, e_{i}\right\rangle .
\end{aligned}
$$


Combining these three formulas, we deduce

(A.5) Proposition. The sectional curvatures of $g=d r^{2}+f^{2}(r) g(r, x)$ for the plane sections $e_{i} \wedge e_{j}$ are given by

$$
\begin{aligned}
K\left(e_{i} \wedge e_{j}\right)= & -\left(\frac{f^{\prime}}{f}\right)^{2}-\left(\frac{f^{\prime}}{f}\right)\left[A\left(\bar{e}_{i}\right)+A\left(\bar{e}_{j}\right)\right]+\left(\frac{1}{f^{2}}-1\right) A\left(\bar{e}_{i}\right) A\left(\bar{e}_{j}\right) \\
& -\left(\frac{1}{f^{2}}-1\right)\left\langle\bar{S}\left(e_{i}\right), e_{j}\right\rangle^{2}+\frac{1}{f^{2}} \bar{K}\left(\bar{e}_{i} \wedge \bar{e}_{j}\right)
\end{aligned}
$$

We also have

(A.6) LeMMA.

$$
\begin{gathered}
\left\langle\nabla_{e_{i}} \nabla_{\partial / \partial r} \frac{\partial}{\partial r}, e_{i}\right\rangle=0 . \\
\left\langle\nabla_{\partial / \partial r} \nabla_{e_{i}} \frac{\partial}{\partial r}, e_{i}\right\rangle=\frac{f^{\prime \prime} f-\left(f^{\prime}\right)^{2}}{f^{2}}+\frac{f^{\prime}}{f}-A\left(\bar{e}_{i}\right)+\left\langle\bar{\nabla}_{\partial / \partial r} \bar{\nabla}_{e_{i}} \frac{\partial}{\partial r}, e_{i}\right\rangle . \\
\left\langle\nabla_{\left[e_{i}, \partial / \partial r\right]} \frac{\partial}{\partial r}, e_{i}\right\rangle=\left(\frac{f^{\prime}}{f}\right)^{2}+\frac{f^{\prime}}{f}-A\left(\bar{e}_{i}\right)+\left\langle\bar{\nabla}_{\left[e_{i}, \partial / \partial r\right]} \frac{\partial}{\partial r}, e_{i}\right\rangle .
\end{gathered}
$$

These three formulas give

(A.7) Proposition. The sectional curvatures of $g=d r^{2}+f^{2}(r) g(r, x)$ corresponding to the plane sections $e_{i} \wedge \partial / \partial r$ are given by

$$
K\left(e_{i} \wedge \frac{\partial}{\partial r}\right)=-\frac{f^{\prime \prime}}{f}-2 \frac{f^{\prime}}{f} A\left(\bar{e}_{i}\right)+\bar{K}\left(\bar{e}_{i} \wedge \frac{\partial}{\partial r}\right)
$$

Choose $\left\{\bar{e}_{i}\right\}$ to be an orthonormal set of principal vectors with principal curvatures $\bar{\lambda}_{i}$, i.e.,

$$
\bar{S}\left(\bar{e}_{i}\right)=\bar{\nabla}_{\bar{e}_{i}} \frac{\partial}{\partial r}=\bar{\lambda}_{i} \bar{e}_{i}
$$

Then $A\left(\bar{e}_{i}\right)=\bar{\lambda}_{i}$ and $\left\langle\bar{S}\left(e_{i}\right), e_{j}\right\rangle=0$ for $i \neq j$. Therefore we have

$$
\begin{gathered}
K\left(e_{i} \wedge e_{j}\right)=-\left(\frac{f^{\prime}}{f}\right)^{2}-\left(\frac{f^{\prime}}{f}\right)\left(\bar{\lambda}_{i}+\bar{\lambda}_{j}\right)+\left(\frac{1}{f^{2}}-1\right) \bar{\lambda}_{i} \bar{\lambda}_{j}+\frac{1}{f^{2}} \bar{K}\left(\bar{e}_{i} \wedge \bar{e}_{j}\right), \\
K\left(e_{i} \wedge e_{0}\right)=-\frac{f^{\prime \prime}}{f}-2 \frac{f^{\prime}}{f} \bar{\lambda}_{i}+\bar{K}\left(\bar{e}_{i} \wedge e_{0}\right) \quad \text { where } e_{0}=\frac{\partial}{\partial r}
\end{gathered}
$$


It follows that for $i \geqslant 1$,

$$
\begin{aligned}
\operatorname{Ric}\left(e_{i}\right)= & \operatorname{Ric}\left(e_{i}, e_{i}\right)=\sum_{\substack{j=0 \\
j \neq 1}}^{m} K\left(e_{i} \wedge e_{j}\right) \\
= & -\frac{f^{\prime \prime}}{f}-(m-1)\left(\frac{f^{\prime}}{f}\right)^{2}-\left(\frac{f^{\prime}}{f}\right) \operatorname{Tr} \bar{S} \\
& -m\left(\frac{f^{\prime}}{f}\right) \bar{\lambda}_{i}+\left(\frac{1}{f^{2}}-1\right) \bar{\lambda}_{i}\left[(\operatorname{Tr} \bar{S})-\bar{\lambda}_{i}\right] \\
& +\left(1-\frac{1}{f^{2}}\right) \bar{K}\left(\frac{\partial}{\partial r} \wedge e_{i}\right)+\frac{1}{f^{2}} \overline{\operatorname{Ric}}\left(\bar{e}_{i}\right), \\
\operatorname{Ric}\left(\frac{\partial}{\partial r}\right)= & -m\left(\frac{f^{\prime \prime}}{f}\right)-2\left(\frac{f^{\prime}}{f}\right) \operatorname{Tr} \bar{S}+\overline{\operatorname{Ric}}\left(\frac{\partial}{\partial r}\right) .
\end{aligned}
$$

From this and the fact that

$$
\bar{\kappa}_{r}=\bar{\kappa}-2 \overline{\operatorname{Ric}}\left(\frac{\partial}{\partial r}\right)+\left(\sum_{\substack{i \neq j \\ i, j=1}} \bar{\lambda}_{i} \bar{\lambda}_{j}\right)
$$

we deduce that

(A.8) Proposition. Suppose $\bar{g}=d r^{2}+g(r, x)$ and $g=d r^{2}+f^{2}(r) g(r, x)$ have scalar curvatures $\bar{\kappa}$ and $\kappa$, respectively. Then

$$
\kappa=-m(m-1)\left(\frac{f^{\prime}}{f}\right)^{2}-2 m \frac{f^{\prime \prime}}{f}-(2 m+2) \frac{f^{\prime}}{f} \operatorname{Tr} \bar{S}+\left(\frac{1}{f^{2}}-1\right) \bar{\kappa}_{r}+\bar{\kappa},
$$

where $\operatorname{Tr} \bar{S}$ is the trace of $\bar{S}=\bar{\nabla}(\partial / \partial r)$, and $\bar{\kappa}_{r}$ is the scalar curvature of the hypersurface $\{r\} \times N$ at distance $r$ to the boundary.

Similar computations give us the following formulas for the deformation $\phi^{2}(r) d r^{2}$ $+g(r, x)$ (also see [20]).

(A.9) Proposition. Let $g^{\prime}=\phi^{2}(r)^{\prime} d r^{2}+g(r, x)$, and let $K^{\prime}$ and $\mathrm{Ric}^{\prime}$ denote the sectional and Ricci curvatures of $g^{\prime}$. Suppose that $\left\{\bar{e}_{i}\right\}$ is an orthonormal set of principal vectors of $\bar{S}=\bar{\nabla}(\partial / \partial r)$ with principal curvatures $\bar{\lambda}_{i}$, and $\partial / \partial s=$ $(1 / \phi)(\partial / \partial r)$. Then

$$
\begin{aligned}
& \operatorname{Ric}^{\prime}\left(\frac{\partial}{\partial s}\right)=\frac{1}{\phi^{2}} \overline{\operatorname{Ric}}\left(\frac{\partial}{\partial r}\right)+\frac{\phi^{\prime}}{\phi^{3}} \operatorname{Tr} \bar{S} \\
\operatorname{Ric}^{\prime}\left(\bar{e}_{i}\right)= & \overline{\operatorname{Ric}}\left(\bar{e}_{i}\right)+\left[\frac{\phi^{\prime}}{\phi^{3}}+\left(1-\frac{1}{\phi^{2}}\right) \operatorname{Tr} \bar{S}\right] \bar{\lambda}_{i} \\
& +\left(\frac{1}{\phi^{2}}-1\right)\left[\bar{K}\left(e_{i} \wedge \frac{\partial}{\partial r}\right)+\bar{\lambda}_{i}^{2}\right] \\
= & \overline{\operatorname{Ric}}\left(\bar{e}_{i}\right)+\frac{\phi^{\prime}}{\phi^{3}} \bar{\lambda}_{i}+\left(1-\frac{1}{\phi^{2}}\right)\left[\overline{\operatorname{Ric}}_{r}\left(\bar{e}_{i}\right)-\overline{\operatorname{Ric}}\left(\bar{e}_{i}\right)\right]
\end{aligned}
$$


where $\overline{\mathrm{Ric}}_{r}$ is the Ricci curvature of the hypersurface $\{r\} \times N$ at distance $r$ to the boundary.

(A.10) Proposition. Suppose $\bar{g}=d r^{2}+g(r, x)$ and $g^{\prime}=\phi^{2}(r) d r^{2}+g(r, x)$ have scalar curvatures $\bar{\kappa}$ and $\kappa^{\prime}$, respectively. Then

$$
\kappa^{\prime}=2 \frac{\phi^{\prime}}{\phi^{3}} \operatorname{Tr} \bar{S}+\frac{1}{\phi^{2}} \bar{\kappa}+\left(1-\frac{1}{\phi^{2}}\right) \bar{\kappa}_{r},
$$

where $\bar{\phi}_{r}$ is the scalar curvature of $\{r\} \times N$.

Combining (A.8) and (A.10), we have the following formula for the scalar curvature $\kappa^{\prime}$ of $g^{\prime}=\phi^{2}(r) d r^{2}+f^{2}(r) g(r, x)$ in terms of $\bar{\kappa}, \bar{\kappa}_{r}$, and $\operatorname{Tr} \bar{S}$ of $\bar{g}=d r^{2}+g(r, x)$.

$$
\begin{aligned}
\kappa^{\prime}= & \frac{-m(m+1)}{\phi^{2}}\left(\frac{f^{\prime}}{f}\right)^{2}-2 m \frac{f^{\prime \prime}}{f} \frac{1}{\phi^{2}}+2 m \frac{\phi^{\prime}}{\phi^{3}} \frac{f^{\prime}}{f} \\
& +\operatorname{Tr} \bar{S}\left(\frac{2 \phi^{\prime}}{\phi^{3}}-\frac{2 m+2}{\phi^{2}} \frac{f^{\prime}}{f}\right)+\left(\frac{1}{f^{2}}-\frac{1}{\phi^{2}}\right) \bar{\kappa}_{r}+\frac{1}{\phi^{2}} \bar{\kappa} .
\end{aligned}
$$

(A.12) ThEOREM. Let $M$ be a manifold with boundary $N^{m}$ and $m=\operatorname{dim} N \geqslant 2$. Suppose that the tubular neighborhood of $N$ is normalized to be of width 1 , i.e., $[0,1] \times N$. If $\bar{\kappa} \geqslant 0, \bar{\kappa}>16 m /(m+1)$ on $[0,1] \times N$, and $\bar{\kappa}_{r} \geqslant 0$, then the metric can be deformed to a complete metric, which ends with the product metric $N \times R$ near infinity, with scalar curvature $\kappa^{\prime} \geqslant 0$.

(A.13) REMARKs. (1) For $m \geqslant 3$, since $m(m+1) \geqslant 16 m /(m+1)$, the condition “ $\bar{\kappa}>16 m /(m+1)$ " can be replaced by " $\bar{\kappa} \geqslant$ the scalar curvature of the standard sphere $S^{m+1}$ of the same dimension".

(2) $\kappa^{\prime}>0$ provided that $\bar{\kappa}>0$ and $\bar{\kappa}_{r}>0$.

Proof of The Theorem. In (A.11) set $\phi=f^{m+1}$. Then

$$
\frac{2 \phi^{\prime}}{\phi^{3}}-\frac{2 m+2}{\phi^{3}} \frac{f^{\prime}}{f}=0,
$$

i.e., the term involving $\operatorname{Tr} \bar{S}$ vanishes. Now we want to find positive smooth function $f$ such that

$$
\begin{aligned}
& f(r) \equiv 0 \text { for } r \geqslant 1 . \\
& \lim _{r \rightarrow 0} f(r)=\infty \text { and } \int_{1}^{r} \phi=\int_{1}^{r} f^{m+1} \text { diverges. } \\
& \frac{-m(m+1)}{\phi^{2}}\left(\frac{f^{\prime}}{f}\right)^{2}-2 m \frac{f^{\prime \prime}}{f} \frac{1}{\phi^{2}}+2 m \frac{\phi^{\prime}}{\phi^{3}} \frac{f^{\prime}}{f}+\frac{1}{\phi^{2}} \bar{\kappa} \geqslant 0 .
\end{aligned}
$$

i.e.,

$$
\frac{1}{f^{2 m+6}}\left\{\kappa\left(m^{2}+3 m\right)\left(\frac{f^{\prime}}{f}\right)^{2}-2 m\left(\frac{f^{\prime \prime}}{f}\right)\right\} \geqslant 0 \quad \text { for } 0<r \leqslant 1
$$


Let $k=$ the minimum of $\bar{\kappa}$ on $[0,1] \times N$ and $f^{\prime} / f=g$. Then it suffices to find a function $g$ satisfying

(a) $g(r) \equiv 0$ for $r \geqslant 1$,

(b) $\lim _{r \rightarrow 0} g(r)=-\infty$,

(c) $k+\left(m^{2}+m\right) g^{2}-2 m g^{\prime} \geqslant 0$.

Condition (b) guarantees that function $f$ increases rapidly when $r$ tends to zero and, hence, satisfies (2).

Let $g=-c / r+c(c>0)$. Then

$$
\begin{aligned}
& k+\left(m^{2}+m\right) g^{2}-2 m g^{\prime} \\
& =\frac{1}{r^{2}}\left\{r^{2}\left(k+c^{2}\left(m^{2}+m\right)\right)-2\left(m^{2}+m\right) c r+\left(m^{2}+m\right) c^{2}-2 m c\right\} \\
& =\frac{1}{r^{2}} h(r), \\
& h(0)=\left(m^{2}+m\right) c^{2}-2 m c \geqslant 0 \quad \text { iff } \quad c \geqslant \frac{2}{m+1}, \\
& h(1)=k-2 m c \geqslant 0 \text { iff } k \geqslant 2 m c .
\end{aligned}
$$

The discriminant of $h(r)<0$ iff

$$
k>\frac{2 c^{2}\left(m^{2}+m\right)}{(m+1) c-2}=k_{0} .
$$

$k_{0}=16 m /(m+1)$ is the srnallest among such numbers if we choose $c=4 /(m+1)$.

This function $g=-c / r+c(c=4 /(m+1))$ satisfies (a)-(c), except that it is not smooth at $r=1$.

If we assume $k>16 m /(m+1)$, then we can smooth out the corner without affecting inequality (c).

Thus $f=\exp \int_{1}^{r} g$ is the function we are seeking.

It is easy to see that the trace of $S=\nabla(\partial / \partial s)$ in this new metric is negative near infinity; therefore, using Theorem (A.2), we can again deform the metric near infinity to the product metric on $N \times R$. Q.E.D.

5. The index theorems. In this section we set up the framework of the heat equation method and derive the index formulas for the Dirac operator. We omit the proofs of most of the standard facts because they are just trivial modifications of the proofs given in $[\mathbf{8}, \mathbf{1 1}]$, which are the main references for this section.

Let $X^{m+1}=C_{0,1}\left(N^{m}\right) \cup M$ be a space with conical singularity. We first assume that the Dirac operator $\bar{D}$ on $X$ is selfadjoint, i.e., the eigenvalues of $\tilde{D}$, the Dirac operator on $N$, are greater than or equal to $1 / 2$. Let $\Delta=\bar{D} * \bar{D}=\bar{D}_{0}^{*} \bar{D}_{0}$ be the Dirac Laplacian. The following facts can be proved by exactly the same arguments as in [8].

(1) The heat kernel of $\Delta, E(u, v, t)=e^{-t \Delta}$ on $X$, is of trace class, i.e.,

$$
\int_{X} E(v, v, t)<\infty .
$$


This implies that Green's operator is compact; therefore, Fredholm theory can be applied to show that the eigenvalues of $\Delta$ on $X$ are discrete and $L^{2}$-completeness of the eigenspinors of $\Delta$ holds.

(2) Let $E_{1}$ and $E_{2}$ denote the heat kernels on $C(N)$ and $M$, respectively. Then for all $N$ there exists $K_{N}$ such that

$$
\left|\int_{C_{0,1}(N)} E(u, u, t)-\int_{C_{0,1}(N)} E_{1}(u, u, t)\right|<K_{N} t^{N},
$$

and, hence,

$$
\int_{X} E(v, v, t)=\int_{C_{0,1}(N)} E_{1}(u, u, t)+\int_{M} E_{2}(y, y, t)+O\left(t^{N}\right) .
$$

Thus the study of the trace of the heat kernel, $\operatorname{tr} E(t)$, is reduced to studying $\int_{C_{0.1}(N)} E_{1}(u, u, t)$, where $E_{1}$ is the heat kernel of the Dirac Laplacian on the cone $C(N)$. Using the conformal homogeneity of $C(N)$, one can again reduce the explicit calculation of the coefficients of the asymptotic expansion of $\int_{C_{0.1}(N)} E_{1}(u, u, t)$ to the calculation of the pointwise coefficients of $\operatorname{tr} E_{1}(t)$ at $r=1$ and to the calculation of a certain global spectral invariant $\psi\left(N^{m}\right)$ of $N^{m}$, which is the contribution from the singularity at the cone tip $\{P\}$.

(3) Let the pointwise asymptotic expansion of $E_{1}((r, x),(r, x), t)$ be $\sum a_{j / 2}(r, x) t^{-(m+1) / 2+j / 2}$, where $(r, x)=u$ is the (polar) coordinate on the cone $C(N)$. Define

$$
a_{j / 2}(1)=\int_{N} a_{j / 2}(1, x)
$$

and

$$
\mu_{k}(t)=\int_{N}\left[\operatorname{tr} E_{1}(1, x, t)-\sum_{j=0}^{k} a_{j / 2}(1, x) t^{-(m+1) / 2+j / 2}\right]
$$

Let

$$
\text { p.f. } \int_{X} \bar{a}_{j / 2}(v, t)
$$

denote the finite part of the (divergent) integral

$$
\int_{X} \bar{a}_{j / 2}(v, t)=\lim _{\varepsilon \rightarrow 0} \int_{X_{\varepsilon}} \bar{a}_{j / 2}(v, t)
$$

where $\bar{a}_{j / 2}(v, t)$ are the coefficients of the pointwise asymptotic expansion of $E(v, v, t)$ on $X$, and $X_{\varepsilon}=X \cup C_{0, \varepsilon}(N)$ (see [8]), i.e.,

$$
\text { p.f. } \int_{X} \bar{a}_{j / 2}=\left\{\begin{array}{l}
\int_{M} \bar{a}_{j / 2}(y)+\frac{1}{m+1-j} \int_{N} a_{j / 2}(1, x) \text { if } j \neq m+1, \\
\int_{M} \bar{a}_{j / 2}(y) \text { if } j=m+1
\end{array}\right.
$$


Then we have

$$
\begin{aligned}
\operatorname{tr} E(t)= & \int_{X} E(v, v, t)-\sum_{j=0}^{k}\left(\text { p.f. } \int_{X} \bar{a}_{j / 2}\right) t^{-(m+1) / 2+j / 2} \\
& -\frac{1}{2} a_{(m+1) / 2}(1) \log t+\psi(N)
\end{aligned}
$$

where

$$
\begin{aligned}
\psi(N)=\frac{1}{2}\left\{\int_{0 N}^{\infty} t^{-1} \operatorname{tr} E_{1}(1, x) d t+\int_{0}^{1} t^{-1} \mu_{k}(t) d t\right. & \\
& \left.+\sum_{\substack{j \neq m+1 \\
j=0}}\left(\frac{a_{j / 2}(1)}{-(m+1) / 2}+\frac{j}{2}\right)\right\}
\end{aligned}
$$

It can be seen that this contribution $2 \psi(N)$ is formally the constant term in the Laurent expansion at $s=0$ of $\Gamma(s) \zeta(s)$ at $r=1$ if the relation between the heat kernel and the zeta function holds, i.e.,

$$
\Gamma(s) \zeta(s)=\int^{\infty} t^{s-1} e^{-t \Delta} d t
$$

as in the compact case. Indeed, this relation will follow from the explicit calculation of the functional calculus of the (Dirac) Laplacian $\Delta$ on the cone $C(N)$ (see [8]).

We now outline the theory of the functional calculus of the Laplacian on cones. For details see [8 and 11]. Recall that if $g$ is a smooth function with compact support in $(0, \infty)$ its Hankel transform is defined by

$$
H_{\nu}(g)(\lambda)=\int_{0}^{\infty} J_{\nu}(\lambda r) g(r) r d r
$$

where $J_{\nu}$ is the Bessel function of order $\nu$ and $\nu>-1$. The Hankel inversion formula (see [12 p. 73; 29]) says that

$$
g(r)=H_{\nu}\left[H_{\nu}(g)\right](r) \text { for } \nu>-1 .
$$

Moreover, we have the Plancherel formula (see [27])

$$
\int_{0}^{\infty}|g(r)|^{2} r d r=\int_{0}^{\infty}\left|H_{\nu}(g)(\lambda)\right|^{2} \lambda d \lambda
$$

Thus $H$ can be extended to an isometry from $L^{2}((0, \infty), r d r)$ to $L^{2}((0, \infty), \lambda d \lambda)$. Define

$$
\Delta_{\mu}^{ \pm} g=-g^{\prime \prime}-\frac{m}{r} g^{\prime}+\left[\mu^{2} \mp \mu-\frac{m^{2}-2 m}{4}\right] \frac{2}{r^{2}} g
$$

the ordinary differential operator in (2.6). In view of (2.6)--(2.9) we have

$$
H_{\nu_{j}^{ \pm}}\left(r^{-c} \Delta_{\mu_{j}}^{ \pm} g\right)=\lambda^{2} H_{\nu_{j}^{ \pm}}\left(r^{-c} g\right)
$$


where $c=(1-m) / 2$ and $\nu_{j}^{ \pm}=12 \mu_{j} \pm 1 \mid / 2$. Set $S_{j}^{ \pm}=2^{-1 / 2}\left(\bar{\phi}_{j} \pm \partial /(\partial r) \bar{\phi}_{j}\right)$, where $\tilde{D} \phi_{j}=\mu_{j} \phi_{j}$ and $\left\|\phi_{j}\right\|_{N}=1$. Then the map $F$ defined by

$$
\begin{aligned}
\phi & =\sum_{j} f_{j}^{+}(r) S_{j}^{+}+f_{j}^{-}(r) S_{j}^{-} \\
& \rightarrow \sum_{j}\left[\lambda^{c} H_{\nu_{j}^{+}}\left(r^{-c} f_{j}^{+}\right)(\lambda) S_{j}^{+}+\lambda^{c} H_{\nu_{j}^{-}}\left(r^{-c} f_{j}^{-}\right)(\lambda) S_{j}^{-}\right]
\end{aligned}
$$

provides an isometry of $L^{2}$ spinors on the $(r, x)$ cone onto $L^{2}$ spinors on the $(\lambda, y)$ cone such that

$$
F(\Delta \theta)=\lambda^{2} F(\theta) \text { for all } \theta \in \operatorname{dom}(\Delta),
$$

i.e., a spectral representation of $\Delta$ while $\Delta$ is carried into multiplication by $\lambda^{2}$. Notice that without the assumption that $\left|\mu_{j}\right| \geqslant 1 / 2$, we must include some $H_{-\nu_{j}^{ \pm}}$in (5.6), (5.7), corresponding to each selfadjoint extension of $\left(D_{0}\right)^{2}$. We discuss this later.

Combining this spectral representation with the Hankel inversion formula, we find that, at least formally, the following relation holds for suitable $f$.

$$
\begin{aligned}
f(\Delta)=\left(r_{1} r_{2}\right)^{c} \sum_{j} \int_{0}^{\infty} f\left(\lambda^{2}\right)[ & J_{\nu_{j}^{+}}\left(\lambda r_{1}\right) J_{\nu_{j}^{+}}\left(\lambda r_{2}\right) S_{j}^{+}\left(x_{1}\right) \otimes S_{j}^{+}\left(x_{2}\right) \\
& \left.+J_{v_{j}^{-}}\left(\lambda r_{1}\right) J_{\nu^{-}}\left(\lambda r_{2}\right) S_{j}^{-}\left(x_{1}\right) \otimes S_{j}^{-}\left(x_{2}\right)\right] \cdot \lambda d \lambda .
\end{aligned}
$$

Notice that in case of $C\left(S_{1}^{m}\right)=R^{m+1}$ the Hankel transform is nothing but the Fourier transform in polar coordinates, and (5.8) is just the representation of the kernel $f(\Delta)$ via the Fourier inversion formula.

The right side should be interpreted as a convergent sum in the distribution sense. It defines families of functions of the Dirac operator $\tilde{D}$ on $N^{m}$ parameterized by $r_{1}, r_{2}$. This crucial observation allows us to bring in the functional calculus of $D$ on $N$ and thereby "sum" the series. We refer to [8 and 11] for a rigorous justification of (5.8) and details of the following discussion of kernels.

For the purpose of calculating the coefficients of the asymptotic expansion of the heat kernel, we need only consider the following examples of $f$.

EXAMPLE 1. The heat kernel $e^{-t \Delta}$, i.e., $f(\lambda)=e^{-t \lambda}$. Using Weber's second exponential integral [29, p. 395], we have

$$
\begin{aligned}
e^{-t \Delta}=\left(r_{1} r_{2}\right)^{c} \sum_{j} \int_{0}^{\infty} e^{-\lambda^{2} t}\left[J_{v_{j}^{+}}\left(\lambda r_{1}\right) J_{\nu_{j}^{+}}\left(\lambda r_{2}\right) S_{j}^{+} \otimes S_{j}^{+}\right. & \left.+J_{\nu_{j}^{-}}\left(\lambda r_{1}\right) J_{\nu_{j}^{-}}\left(\lambda r_{2}\right) S_{j}^{-} \otimes S_{j}^{-}\right] \lambda d \lambda \\
=\left(r_{1} r_{2}\right)^{c} \sum_{j} \frac{1}{2 t} e^{-\left(r_{1}^{2}+r_{2}^{2}\right) / 4 t} & \\
& \times\left[I_{\nu_{j}^{+}}\left(\frac{r_{1} r_{2}}{2 t}\right) S_{j}^{+} \otimes S_{j}^{+}+I_{\nu_{j}^{-}}\left(\frac{r_{1} r_{2}}{2 t}\right) S_{j}^{-} \otimes S_{j}^{-}\right]
\end{aligned}
$$

where $I_{\nu}$ is the modified Bessel function of order $\nu$. 
The elliptic estimates, together with the asymptotic expansion of $I_{\nu}$, show that (5.9) converges uniformly on compact subsets of $R^{+} \times C(N) \times C(N)$.

EXAMPle 2. The zeta function $\Gamma(s) \Delta^{-s}$, i.e., $f(\lambda)=\Gamma(s) \lambda^{-s}$. This is given by the Weber-Schafheitlin integral [29, p. 401]

$$
\begin{aligned}
\Gamma(s) & \int_{0}^{\infty} \lambda^{-2 s} J_{\nu}\left(\lambda r_{1}\right) J_{\nu}\left(\lambda r_{2}\right) \lambda d \lambda \\
& =\frac{r_{1}^{\nu} r_{2}^{-\nu+2(s-1)} \Gamma(\nu-s+1)}{2^{2 s-1} \Gamma(\nu+1)} F\left(1-s+\nu, s+1, \nu+1, r_{1}^{2} / r_{2}^{2}\right)
\end{aligned}
$$

if $r_{1}<r_{2}$ and $\nu>s-1>-1 / 2$, where $F$ is the hypergeometric function.

We are primarily interested in the trace of the corresponding kernel at $r_{1}=r_{2}=1$. In this case (5.10) is reduced to

$$
\frac{1}{2 \sqrt{\pi}} \frac{\Gamma(\nu-s+1)}{\Gamma(\nu+s)} \Gamma\left(s-\frac{1}{2}\right)
$$

(see [21, p. 243]), and the kernel is given by

$$
\begin{aligned}
\Gamma(s) \zeta(s) & =\left.\Gamma(s) \Delta^{-s}\right|_{r_{1}=r_{2}=1} \\
& =\sum_{j} \frac{\Gamma\left(s-\frac{1}{2}\right)}{2 \sqrt{\pi}}\left[\frac{\Gamma\left(\nu_{j}^{+}-s+1\right)}{\Gamma\left(\nu_{j}^{+}+s\right)} S_{j}^{+} \otimes S_{j}^{+}+\frac{\Gamma\left(\nu_{j}^{-}-s+1\right)}{\left(\nu_{j}^{-}+s\right)} S_{j}^{-} \otimes S_{j}^{-}\right] .
\end{aligned}
$$

It follows from a careful examination of (5.9) and (5.11) that a relation similar to (5.4) holds, and we can compute the coefficients of the asymptotic expansion of the heat kernel in terms of the residues of the zeta function $\Gamma(s) \zeta(s)$ at simple poles; see [8] for the precise statements and proofs. Thus, the contribution to the constant term from the singularity $\psi(N)$ is indeed the constant term of the Laurent expansion of $\Gamma(s) \zeta(s)$ at $s=0$, i.e.,

$$
\psi(N)=\left.\frac{1}{2} \frac{d}{d s}[s \Gamma(s) \zeta(s)]\right|_{s=0} .
$$

To compute this term explicitly, we need the following lemma from [8]. Let $B_{l}$ denote the $l$ th Bernoulli number, and let $C_{l}=(-1)^{l-1} B l / l$.

(5.13) LEMMA.

$$
\begin{aligned}
& \frac{\Gamma(\nu-s)}{\Gamma(\nu+s)} \sim \nu^{-2 s}\left(1+\frac{s}{\nu}+s \sum_{l=1}^{\infty} C_{l} \nu^{-2 l}\right)+O\left(s^{2}\right) \text { as } \nu \rightarrow \infty \\
& \frac{\Gamma(\nu-s+1)}{\Gamma(\nu+s)} \sim \nu^{1-2 s}\left(1+s \sum_{l=1} C_{l} \nu^{-2 l}\right)+O\left(s^{2}\right) \text { as } \nu \rightarrow \infty .
\end{aligned}
$$

Now we are ready to compute the index of $D^{+}$on the even-dimensional space $X^{m+1}=C_{0,1}\left(N^{m}\right) \cup M$. Assume that $m+1=2 k$. Recall that the bundle of spinors $S$ on $X$ splits into $S^{+} \oplus S^{-}$, where $S^{+}$and $S^{-}$are the two irreducible bundles 
corresponding to the two irreducible spin-representations of $\operatorname{Spin}(2 k)$. Since $N^{m}$ has the induced spin structure, $\left.S\right|_{N}$ splits into two nonisomorphic modules $T^{+}$and $T^{-}$ over $\mathrm{Cl}(2 k-1)$ corresponding to the $(+)$ and $(-)$ eigenspaces of multiplication by the volume form $\omega_{2 k-1}=i^{k} e_{1} e_{2}, \ldots, e_{2 k-1}$ on $N^{m}$, respectively, where $i=\sqrt{-1}$ and $\left\{e_{i}\right\}$ is an orthonormal basis on $N$. Note that $T^{+}$and $T^{-}$are isomorphic irreducible bundles of spinors coming from the two isomorphic spin representations of $\operatorname{Spin}(2 k-1)$. Let $T^{+}$be the irreducible bundle of spinors on $N^{m}$, i.e., $T^{+}=S(N)$ in the notation of $\S 2$. Then the embedding of $S(N)$ into $S(C(N))$ is given by the inclusion $\left.T^{+} \subset S\right|_{N}=T^{+} \oplus T^{-}$and $\left.S\right|_{N}=T^{+} \oplus\left(\partial / \partial r \cdot T^{+}\right)$. To fix the orientation we define the volume form on $C(N)$ to be $\omega_{2 k}=i^{k}(\partial / \partial r) e_{1} e_{2}, \ldots, e_{2 k-1}$. Then it is easy to check that if $\left\{\phi_{i}\right\}$ is a local spinor basis of $S(N)=T^{+}$, then

$$
\left\{\frac{1}{\sqrt{2}}\left(\bar{\phi}_{i}+\frac{\partial}{\partial r} \bar{\phi}_{i}\right)\right\} \text { and }\left\{\frac{1}{\sqrt{2}}\left(\bar{\phi}_{i}-\frac{\partial}{\partial r} \bar{\phi}_{i}\right)\right\}
$$

are the local orthonormal bases of $S^{+}$and $S^{-}$on the cone $C_{0,1}(N)$, respectively.

Let $\bar{D}^{ \pm}, \Delta^{ \pm}$denote $\bar{D}, \Delta$ restricted to $S^{ \pm}$. Then the heat equation method of computing the index (see [1]) gives

$$
\begin{aligned}
\operatorname{Index}\left(\bar{D}^{+}\right)= & \operatorname{dim} \operatorname{Ker}\left(\bar{D}^{+}\right)-\operatorname{dim} \operatorname{Ker}\left(\bar{D}^{-}\right) \\
= & \operatorname{tr}\left(e^{-t \Delta^{+}}\right)-\operatorname{tr}\left(e^{-t \Delta^{-}}\right)=a_{k}^{+}-a_{k}^{-} \\
= & \text {the constant term } a_{k} \text { in the asymptotic expansion } \\
& \text { of } \operatorname{tr}\left(\omega_{2 k} \cdot e^{-t \Delta}\right),
\end{aligned}
$$

where $\omega_{2 k}$ is the volume form on $X^{2 k}$ and - is the module multiplication with respect to the first variable of the heat kernel $e^{-t \Delta}=E(u, v, t)$.

According to (5.3), one easily sees that $a_{k}$ consists of two terms. The first term is the integral of the same local contribution from the interior as in the smooth case. By a theorem of Gilkey [1] this is the integral of the $\hat{A}$-polynomial of Pontrjagin forms $P_{i}$ over $M, \int_{M} \hat{A}(P)$, which is equal to $\int_{X} \hat{A}(P)$ since $\left.\hat{A}\right|_{C_{0,1}(N)} \equiv 0$.

We now compute the second contribution $\psi(N)$. From (5.11), (5.12), it follows that

$$
\psi(N)=\left.\frac{1}{2} \int_{N} \frac{d}{d s}\left[s\left(\Gamma(s) \omega_{2 k} \cdot \zeta(s)\right)\right]\right|_{s=0}
$$

where

$$
\begin{aligned}
\Gamma(s) & \omega_{2 k} \cdot \zeta(s) \\
& =\frac{\sum}{j} \frac{\Gamma\left(s-\frac{1}{2}\right)}{2 \sqrt{\pi}}\left[\frac{\Gamma\left(\nu_{j}^{+}-s+1\right)}{\Gamma\left(\nu_{j}^{+}+s\right)} S_{j}^{+} \otimes S_{j}^{+}-\frac{\Gamma\left(\nu_{j}^{-}-s+1\right)}{\Gamma\left(\nu_{j}^{-}+s\right)} S_{j}^{-} \otimes S_{j}^{-}\right]
\end{aligned}
$$


and $\nu_{j}^{ \pm}=\left|2 \mu_{j} \pm 1\right| / 2$. Using (5.13) and the assumption that $\left|\mu_{j}\right| \geqslant 1 / 2$, we obtain

$$
\begin{aligned}
& \psi(N)=-\left.\frac{1}{2} \frac{d}{d s}\right|_{s=0} s \cdot\{\left.\sum \nu_{j}^{+^{1-2 s}}+s \sum_{l=1}^{\infty} C_{l} \nu_{j}^{+^{-2 l-2 s+1}}\right) \\
&\left.--\sum\left(\nu_{j}^{-1-2 s}+s \sum_{l=1}^{\infty} C_{l} \nu_{j}^{-2 l-2 s+1}\right)\right\} \\
&=-\left.\frac{1}{2} \frac{d}{d s}\right|_{s=0} s \cdot\left\{\sum_{\mu_{j} \geqslant 1 / 2}\left[\left(\mu_{j}-\frac{1}{2}\right)^{1-2 s}+s \sum_{l=1}^{\infty} C_{l}\left(\mu_{j}-\frac{1}{2}\right)^{-2 l-2 s+1}\right]\right. \\
&+\sum_{\mu_{j} \leqslant-1 / 2}\left[\left(\frac{1}{2}-\mu_{j}\right)^{1-2 s}+s \sum_{l=1}^{\infty} C_{l}\left(\frac{1}{2}-\mu_{j}\right)^{-2 l-2 s+1}\right] \\
& \quad-\sum_{\mu_{j} \geqslant 1 / 2}\left[\left(\mu_{j}+\frac{1}{2}\right)^{1-2 s}+s \sum_{l=1}^{\infty} C_{l}\left(\mu_{j}+\frac{1}{2}\right)^{-s l-2 s+1}\right] \\
&\left.-\sum_{\mu_{j} \geqslant-1 / 2}\left[\left(-\mu_{j}-\frac{1}{2}\right)^{1-2 s}+s \sum_{l=1}^{\infty} C_{l}\left(-\mu_{j}-\frac{1}{2}\right)^{-2 l-2 s+1}\right]\right\} .
\end{aligned}
$$

Applying the binomial theorem gives

$$
\left(\mu-\frac{1}{2}\right)^{1-2 s}-\left(\mu+\frac{1}{2}\right)^{1-2 s} \sim-(1-2 s) \mu^{-2 s}+\sum_{k=1}^{\infty} a_{k}(s) s \mu^{-2 s-2 k}
$$

where $a_{k}(s)$ is a polynomial in $s$. Therefore,

$$
\begin{aligned}
\psi(N) & =\left.\frac{1}{2} \frac{d}{d s}\right|_{s=0}\left\{\sum_{\mu_{j} \geqslant 1 / 2}\left[s(1-2 s) \mu_{j}^{-2 s}\right]-\sum_{\mu_{j} \leqslant-1 / 2}\left[s(1-2 s) \mu_{j}^{-2 s}\right]\right\} \\
& =\left.\frac{1}{2} \frac{d}{d s}\right|_{s=0}[s \eta(s)]=\frac{\eta(0)}{2},
\end{aligned}
$$

where

$$
\eta(s)=\sum_{\mu_{j} \neq 0}\left(\operatorname{sign} \mu_{j}\right)\left|\mu_{j}\right|^{-s} .
$$

This is because $\eta(s)$ is holomorphic for $\operatorname{Re}(s)>-1 / 2$; see [3 and 8].

Thus, we can summarize what we have obtained as follows.

(5.18) THEOREM. Let $X^{2 k}=C_{0,1}(N) \cup M$ be a space with a conical singularity. Assume that $\left|\mu_{j}\right| \geqslant 1 / 2$, where $\left\{\mu_{j}\right\}$ are the eigenvalues of $\tilde{D}$ on $N$; i.e., $\bar{D}$ is selfadjoint. Then

$$
\operatorname{Index}\left(\bar{D}^{+}\right)=\int_{X} \hat{A}(p)+\frac{\eta(0)}{2},
$$

where $\hat{A}$ is the Hirzebruch $\hat{A}$-polynomial of Pontrjagin forms $P=\left\{P_{i}\right\}$. 
Although this index formula is equivalent to the one obtained by Atiyah, Patodi, and Singer [3] for manifolds with boundary, we wish to emphasize that (5.18) is the natural index formula for a class of compact singular spaces.

We now treat the general case without assuming $\left|\mu_{j}\right|>1 / 2$. Note that we already had three closed extensions of $D_{0}: \bar{D}_{0}, \bar{D}_{b}$, and $\bar{D}$, where $\bar{D}_{b}$ is the selfadjoint extension under the ideal boundary condition (3.4). Before computing the indices of these operators, we first examine the domains of $\bar{D}_{0}^{*} \bar{D}_{0}, \bar{D}_{b}^{*} \bar{D}_{b}$, and $\bar{D} * \bar{D}$.

(5.19) (1) The domain of $\bar{D}_{0}^{*} \bar{D}_{0}=\Delta_{D}$ contains all the positive solutions (2.10), (2.11), (2.14), (2.16), because $\left\langle\bar{D}^{*} \alpha, \phi\right\rangle=\langle\alpha, \bar{D} \phi\rangle$ for all $\phi \in \operatorname{dom}(\bar{D})$ and all positive solutions $\alpha$, and $\bar{D}^{*}=\bar{D}_{0}$. Moreover, no negative solution is in $\operatorname{dom}\left(\bar{D}_{0}\right)$ or $\operatorname{dom}\left(\Delta_{D}\right)$ by $(2.32)$.

(2) For $\bar{D}_{b}^{*} \bar{D}_{b}=\Delta_{b}$, it follows from (2.11), (2.19), (2.22), (3.4) that the negative solution $r^{(m+1) / 2} J_{-\nu_{j}^{+}}$corresponding to $0<\mu_{j}<1 / 2$ and $r^{(-m+1) / 2} J_{-\nu_{j}^{-}}$corresponding to $-1 / 2<\mu_{j}<0$ are in $\operatorname{dom}\left(\Delta_{b}\right)$, but the positive solutions $r^{(-m+1) / 2} J_{\nu_{j}^{+}}$for $0 \leqslant \mu_{j}<1 / 2$ and $r^{(-m+1) / 2} J_{\nu_{j}^{-}}$for $-1 / 2<\mu_{j} \leqslant 0$ are not.

(3) For $\bar{D} * \bar{D}=\Delta_{N}$ it also follows from (2.19), (2.22) that the negative solutions $r^{(-m+1) / 2} J_{-\nu_{j}^{+}}$and $r^{(-m+1) / 2} J_{-\nu_{j}^{-}}$for $\left|\mu_{j}\right|<1 / 2$ are in $\operatorname{dom}\left(\Delta_{N}\right)$, but the positive solutions $r^{(-m+1) / 2} J_{\nu_{j}^{+}}$and $r^{\left(-m^{j}+1\right) / 2} J_{\nu_{j}^{-}}$for $\left|\mu_{j}\right|<1 / 2$ are not.

For the functional calculus of the operators $\Delta_{D}, \Delta_{b}$, and $\Delta_{N}$, we must include or exclude $J_{ \pm \nu_{j}^{ \pm}}$in (5.7)-(5.11) according to (5.19). Applying the heat equation method to compute the indices as in (5.14), we have

$$
\begin{aligned}
& \operatorname{Index}\left(\bar{D}_{0}^{+}\right)=\operatorname{tr}\left(e^{-t \Delta_{D}^{+}}\right)-\operatorname{tr}\left(e^{-t \Delta_{N}^{-}}\right) . \\
& \operatorname{Index}\left(\bar{D}_{b}^{+}\right)=\operatorname{tr}\left(e^{-t \Delta_{b}^{+}}\right)-\operatorname{tr}\left(e^{-t \Delta_{b}^{-}}\right) . \\
& \operatorname{Index}\left(\bar{D}^{+}\right)=\operatorname{tr}\left(e^{-t \Delta_{N}^{+}}\right)-\operatorname{tr}\left(e^{-t \Delta_{D}^{-}}\right) .
\end{aligned}
$$

Simple calculations similar to (5.15)-(5.17) with appropriate $\pm \nu_{j}^{ \pm}$for $\left|\mu_{j}\right|<1 / 2$ give us the following theorem.

(5.23) THEOREM.

$$
\operatorname{Index}\left(\bar{D}_{0}^{+}\right)=\int_{X} \hat{A}(p)+\frac{\eta(0)-h}{2}-\sum_{0<\mu_{j}<1 / 2} \operatorname{dim}\left(E_{\mu_{j}}\right),
$$

where $h=\operatorname{dim} \operatorname{Ker}(\tilde{D})$, and $E_{\mu_{j}}$ is the eigenspace of $\tilde{D}$ with eigenvalue $\mu_{j}$.

$$
\begin{gathered}
\operatorname{Index}\left(\bar{D}_{b}^{+}\right)=\int_{X} \hat{A}(p)+\frac{\eta(0)}{2} . \\
\operatorname{Index}\left(\bar{D}^{+}\right)=\int_{X} \hat{A}(p)+\frac{\eta(0)+h}{2}+\sum_{-1 / 2<\mu_{j}<0} \operatorname{dim}\left(E_{\mu_{j}}\right) .
\end{gathered}
$$

Note that

$$
\operatorname{Index}\left(\bar{D}^{+}\right)-\operatorname{Index}\left(\bar{D}_{0}^{+}\right)=\sum_{\left|\mu_{j}\right|<1 / 2} \operatorname{dim}\left(E_{\mu_{j}}\right)
$$

is also a trivial consequence of (2.21) and (5.19). 
Combining (4.3) with (5.18), we thus have

(5.24) THEOREM. Suppose $\kappa \geqslant 0$ and $\kappa>0$ somewhere. Then

$$
\operatorname{Index}\left(\bar{D}^{+}\right)=0=\int_{X} \hat{A}(p)+\frac{\eta(0)}{2} .
$$

(5.25) REMARK. If we consider spinors with coefficients in a bundle $E^{k}$ which is (locally) flat in a neighborhood of the singularity at the cone tip, then from $[1,4]$, it is easy to see that the index formula because

$$
\operatorname{Index}\left(\bar{D}^{+}\right)=\int_{X} \operatorname{Ch}(E) \cdot \hat{A}+\frac{\eta(0)}{2} \text { if }\left|\mu_{j}\right| \geqslant \frac{1}{2},
$$

where $\mathrm{Ch}(E)=k+\mathrm{Ch}_{1}(E)+\mathrm{Ch}_{2}(E)+\cdots$ is the Chern character of $E$. Notice that here both $\eta(0)$ and $\left\{\mu_{j}\right\}$ belong to the Dirac operator $\tilde{D}$ on spinors with coefficients in the bundle $\left.E\right|_{N}$.

We now consider the relative index of generalized Dirac oprerators on spinors with coefficients in a hermitian bundle $E^{k}$ which is trivial and flat in a neighborhood of $\{p\}$; i.e., $\left.E^{k}\right|_{C_{0 . u}(N)} \cong C^{k} \times C_{0, u}(N)$ and the curvature $R^{E}$ satisfies $\left.R^{E}\right|_{C_{0 . u}(N)} \equiv 0$.

Let $D_{1}$ and $D_{2}$ denote the (generalized) Dirac operators on sections of the bundles $S(X) \otimes E$ and $S(X) \otimes C^{k}$, respectively. Define the relative index of $\bar{D}_{1}$ and $\bar{D}_{2}$ to be

$$
\operatorname{Ind}\left(\bar{D}_{1}^{+}, \bar{D}_{2}^{+}\right)=\operatorname{Index}\left(\bar{D}_{1}^{+}\right)-\operatorname{Index}\left(\bar{D}_{2}^{+}\right)
$$

Similarly,

$$
\operatorname{Ind}\left(\bar{D}_{1,0}^{+}, \bar{D}_{2,0}^{+}\right)=\operatorname{Index}\left(\bar{D}_{1,0}^{+}\right)-\operatorname{Index}\left(\bar{D}_{2,0}^{+}\right)
$$

Then it follows from (5.23) and (5.25) that

$$
\operatorname{Ind}\left(\bar{D}_{1}^{+}, \bar{D}_{2}^{+}\right)=\operatorname{Ind}\left(\bar{D}_{1,0}^{+}, \bar{D}_{2,0}^{+}\right)=\int_{X} \widehat{\operatorname{Ch}}(E) \hat{A},
$$

where $\widehat{\operatorname{Ch}}(E)=\mathrm{Ch}_{1}(E)+\mathrm{Ch}_{2}(E)+\cdots=\mathrm{Ch}(E)-k$ is the reduced Chern character.

The notion of relative index was first exploited by Gromov and Lawson [18] for complete manifolds with uniformly positive curvature condition at infinity. Our notion (5.26) is called the analytical relative index in [18], and (5.27) actually shows that it is equal to the topological relative index (see [18]) by way of the (absolute) index formulas (5.23) in our case. Notice that we have not yet assumed any positive curvature condition near the cone tip.

Now, in view of (4.5), we can deduce the following result from (5.27). Let $\sigma \in \Gamma(S(X))$ and $\sigma \in \Gamma(E)$. Put

$$
R_{0}(\sigma \otimes e)=\frac{1}{2} \sum_{j, k}\left(e_{j} e_{k} \sigma\right) \otimes R_{e_{j} e_{k}}^{E}(e),
$$

as in (1.11) and (1.12).

(5.28) THEOREM. Suppose that on $X=C_{0,1}(N) \cup M^{2 k}, \kappa \geqslant 4\left\|R_{0}\right\|$ and $\kappa>4\left\|R_{0}\right\|$ somewhere. Then $\int_{X} \widehat{\mathrm{Ch}}(E) \cdot \hat{A}=0$.

This serves as a topological obstruction to the existence of such a metric. Notice that $\left.\widehat{\mathrm{Ch}}(E)\right|_{C_{0,1}(N)} \equiv 0$ since $\left.R^{E}\right|_{C_{0, u}(N)} \equiv 0$. 
We can also define the notion of enlargeability and obtain results similar to those in [18].

(5.29) Definition. A Riemannian $n$-manifold $X^{n}$ (possibly incomplete) is enlargeable if $\forall \varepsilon>0$ there exists a finite spin covering manifold $\tilde{X} \rightarrow X$ and an $\varepsilon$-contracting map $\tilde{X} \rightarrow S^{n}$ which is constant outside a compact subset and of nonzero degree.

Here the assumption on the finiteness of the covering is essential for preserving the type of singularities after passing to the covering space. One easily sees that the same untwisting trick of Gromov and Lawson [17] can be applied to prove the following theorem.

(5.30) Theorem. Suppose that in $X=C_{0,1}(N) \cup M^{2 k}, M$ is even dimensional and the interior of $M$ is enlargeable. Then there exists no metric, which is conical near the singularity at the cone tip, with scalar curvature $\kappa$ such that $\kappa \geqslant 0$ and $\kappa>0$ on the interior of $M$.

Proof. We proceed exactly as in the proof of Theorem 3.1 in [17]. Assume that $\kappa$ satisfies $\kappa \geqslant 0$ and $\kappa>0$ on $Y$, the interior of $M$. Since $Y$ is enlargeable, for any $\varepsilon>0$ there exist a finite spin cover $\tilde{Y} \rightarrow Y$ and an $\varepsilon$-contracting map of nonzero degree $f: \tilde{Y} \rightarrow S^{2 k}$ which is constant outside a compact subset $Z \subset \tilde{Y}$. Choose a complex vector bundle $E_{0}$ over $S^{2 k}$ such that $C_{k}\left(E_{0}\right) \neq 0$, where $C_{k}$ is the topdimensional Chern class. Let $\tilde{X}$ denote the space formed by attaching cones to $\tilde{Y}$ along its boundary. Notice that the metric near the boundary of $\tilde{Y}$ can be smoothly extended to an attached cone because $\tilde{Y}$ is a finite cover of $Y$ and has the induced metric from $Y$. Then the map $f$ can be extended to $\tilde{X}$ by sending the attached cones to the same constant point. Let $f^{*} E_{0}=E$ be the pullback of $E_{0}$ by $f$ with the induced connection. If $\varepsilon$ is small enough, then $\kappa>4\left\|R_{0}\right\|$ on $Z$ and, hence, $\kappa \geqslant 4\left\|R_{0}\right\|$ on $\tilde{X}$. It follows from (5.28) that

$$
\begin{aligned}
0 & =\int_{\tilde{X}} \widehat{\mathrm{Ch}}(E) \cdot \hat{A}=\frac{1}{(m-1) !} \int_{\tilde{X}} f^{*} C_{k}\left(E_{0}\right) \\
& =\frac{1}{(m-1) !} \operatorname{deg}(f) \int_{S^{2 k}} C_{k}\left(E_{0}\right) \neq 0 .
\end{aligned}
$$

This is a contradiction. Q.E.D.

\section{REFERENCES}

1. M. F. Atiyah, R. Bott and V. K. Patodi, On the heat equation and the index theorem, Invent. Math. 19 (1973), 279-330.

2. M. F. Atiyah, R. Bott and A. A. Shapiro, Clifford modules, Topology 3 (Suppl. 1) (1964), 3-38.

3. M. F. Atiyah, V. K. Patodi, and I. Singer, Spectral asymmetry and riemannian geometry, I, Math. Proc. Cambridge Philos. Soc. 77 (1975), 43-69.

4. M. F. Atiyah, and I. Singer, The index of elliptic operators. III, Ann. of Math. (2) 87 (1968), 546-604.

5. J. Cheeger, Analytic torsion and the heat equation, Ann. of Math. (2) 109 (1979), 259-322.

6. On the spectral geometry of spaces with cone-like singularities, Proc. Nat. Acad. Sci. U.S.A. 76 (1979), 2103-2106. (A revised version is in preprint (1980).)

7. $\ldots$, On the Hodge theory of riemannian pseudomanifolds, Proc. Sympos. Pure Math., vol. 36, Amer. Math. Soc. Providence, R.I., 1980, pp. 91-145. 
8. __ Spectral geometry of singular riemannian spaces, J. Differential Geom. 18 (1983), 575-657.

9. J. Cheeger and D. Ebin, Comparison theorems in Riemannian geometry, North-Holland, 1975.

10. J. Cheeger, M. Goresky and R. MacPherson, $L^{2}$-cohomology and intersection homology of singular algebraic varieties, Seminar on Differential Geometry, Ann. of Math. Stud., no. 102, Princeton Univ. Press, Princeton, N.J., 1982, pp. 303-340.

11. J. Cheeger and M. Taylor, On the diffraction of waves by conical singularities. I, Comm. Pure Appl. Math. 35 (1982), 275-331.

12. A. Erdelyi (Editor), Higher transcendental functions (Bateman Manuscript Project), Vol. 2, McGraw-Hill, 1953.

13. T. Friedrich, Der erste eigenwert des Dirac-operators einer kompakten riemannschen mannigfaltigkeit nichnegativer skalarkrümmung, Universität zu Berlin (preprint).

14. K. O. Friedrich, The identity of weak and strong extensions of differential operators, Trans. Amer. Soc. 55 (1944), 132-151.

15. M. Gaffney, A special Stoke's theorem for riemannian manifolds, Ann. of Math. (2) 60 (1954), $140-145$.

16. M. Gromov and H. B. Lawson, Spin and the scalar curvature in the presence of a fundamental group. I, Ann. of Math (2) 111 (1980), 209-230.

17. The classification of simply connected manifolds of positive scalar curvature, Ann. of Math. (2) $111(1980), 423-434$.

18. , Positive scalar curvature and the Dirac operator on complete riemannian manifolds, I.H.E.S. Publ. Math. 58 (1983), 83-196.

19. E. L. Ince, Ordinary differential equations, Dover, New York, 1956.

20. P. M. Ingram, Extension aspects and new examples of positively Ricci curved manifolds, Ph.D. Thesis, State Univ. of New York at Stony Brook, Stony Brook, N. Y., 1981.

21. N. Lebedev, Special functions and their applications, Dover, 1972.

22. H. B. Lawson and M. L. Michelsohn, The geometry of spinors (to appear).

23. A. Lichnerowicz, Spineurs harmoniques, C. R. Acad. Sci. Paris Ser. A-B 257 (1963), 7-9.

24. J. Milnor, Remarks concerning spin manifolds, Differential and Combinatorial Topology, Princeton Univ. Press, Princeton, N.J., 1964, pp. 55-62.

25. M. Reed and B. Simon, Functional analysis, methods of modern mathematical physics, Vol. 1, Academic Press, 1972.

26. G. deRham, Variétés différentiables, Hermann, Paris, 1960.

27. I. Sneddon, The use of integral transforms, McGraw-Hill, 1972.

28. I. Stakgold, Boundary value problems of mathematical physics, Vol. 1, Macmillan, 1967.

29. G. Watson, A treatise on the theory of Bessel functions, Cambridge Univ. Press, 1973.

Department of Mathematics, Clark University, Worcester, MassachusetTs 01610

School of Mathematics, The Institute of AdVanced Study, Princeton, New Jersey 08540 